Noname manuscript No.

(will be inserted by the editor)

\title{
Constraining Gas Motions in the Intra-Cluster Medium
}

\author{
Aurora Simionescu • John ZuHone • \\ Irina Zhuravleva • Eugene Churazov • \\ Massimo Gaspari • Daisuke Nagai . \\ Norbert Werner • Elke Roediger . \\ Rebecca Canning • Dominique Eckert • \\ Liyi Gu · Frits Paerels
}

Received: date / Accepted: date

\author{
Aurora Simionescu \\ SRON, Netherlands Institute for Space Research, Sorbonnelaan 2, 3584 CA Utrecht, The \\ Netherlands; E-mail: a.simionescu@sron.nl \\ Institute of Space and Astronautical Science (ISAS), JAXA, 3-1-1 Yoshinodai, Chuo-ku, \\ Sagamihara, Kanagawa, 252-5210, Japan \\ John ZuHone \\ Harvard-Smithsonian Center for Astrophysics, 60 Garden St., Cambridge, MA 02138, USA \\ Irina Zhuravleva \\ Department of Astronomy \& Astrophysics, University of Chicago, 5640 S Ellis Ave, Chicago, \\ IL 60637, USA \\ Kavli Institute for Particle Astrophysics and Cosmology, Stanford University, 452 Lomita \\ Mall, Stanford, CA 94305-4085, USA \\ Department of Physics, Stanford University, 382 Via Pueblo Mall, Stanford, CA 94305-4085, \\ USA
}

Eugene Churazov

Max Planck Institute for Astrophysics, Karl-Schwarzschild-Strasse 1, D-85741 Garching, Germany

Space Research Institute (IKI), Profsoyuznaya 84/32, Moscow 117997, Russia

\section{Massimo Gaspari}

Einstein and Spitzer Fellow, Department of Astrophysical Sciences, Princeton University, 4 Ivy Lane, Princeton, NJ 08544-1001, USA

Daisuke Nagai

Department of Physics, Yale University, PO Box 208101, New Haven, CT, USA

Yale Center for Astronomy and Astrophysics, PO Box 208101, New Haven, CT, USA

Norbert Werner

MTA-Eötvös Loránd University Lendület Hot Universe Research Group, H-1117 Pázmány Péter sétańy 1/A, Budapest, Hungary

Department of Theoretical Physics and Astrophysics, Faculty of Science, Masaryk University, Kotlárská 2, Brno, 61137, Czech Republic

School of Science, Hiroshima University, 1-3-1 Kagamiyama, Higashi-Hiroshima 739-8526, Japan

This is a post-peer-review, pre-copyedit version of an article published in Space Science Reviews. The final authenticated version is available online at: https://doi.org/10.1007/s11214-019-0590-1 
Abstract The detailed velocity structure of the diffuse X-ray emitting intracluster medium (ICM) remains one of the last missing key ingredients in understanding the microphysical properties of these hot baryons and constraining our models of the growth and evolution of structure on the largest scales in the Universe. Direct measurements of the gas velocities from the widths and shifts of X-ray emission lines were recently provided for the central region of the Perseus Cluster of galaxies by Hitomi, and upcoming high-resolution Xray microcalorimeters onboard XRISM and Athena are expected to extend these studies to many more systems. In the mean time, several other direct and indirect methods have been proposed for estimating the velocity structure in the ICM, ranging from resonant scattering to X-ray surface brightness fluctuation analysis, the kinematic Sunyaev-Zeldovich effect, or using optical line emitting nebulae in the brightest cluster galaxies as tracers of the motions of the ambient plasma. Here, we review and compare the existing estimates of the velocities of the hot baryons, as well as the various overlapping physical processes that drive motions in the ICM, and discuss the implications of these measurements for constraining the viscosity and identifying the source of turbulence in clusters of galaxies.

Keywords Clusters of galaxies · Intracluster medium · X-ray spectroscopy • Large-scale structure

Elke Roediger

E.A. Milne Centre for Astrophysics, Department of Physics and Mathematics, University of Hull, Hull, HU6 7RX, UK

Rebecca Canning

Kavli Institute for Particle Astrophysics and Cosmology, Stanford University, 452 Lomita Mall, Stanford, CA 94305-4085, USA

Department of Physics, Stanford University, 382 Via Pueblo Mall, Stanford, CA 94305-4085, USA

Dominique Eckert

Max-Planck-Institut für extraterrestrische Physik, Giessenbachstrasse 1, 85748 Garching, Germany

Department of Astronomy, University of Geneva, Ch. dEcogia 16, CH-1290 Versoix, Switzerland

Liyi Gu

RIKEN High Energy Astrophysics Laboratory, 2-1 Hirosawa, Wako, Saitama 351-0198, Japan

SRON Netherlands Institute for Space Research, Sorbonnelaan 2, 3584 CA Utrecht, the Netherlands

Frits Paerels

Columbia Astrophysics Laboratory and Department of Astronomy, Columbia University, New York, NY, USA 


\section{Introduction and motivation}

The evolution of structure in the Universe is a dynamical process. Both on the largest scales, where mergers between clusters of galaxies and accretion from the surrounding cosmic web drive the growth of the most massive haloes, and on smaller scales, where supermassive black holes (SMBHs) are intimately interconnected with the evolution of their host galaxies, information about the kinematics associated with these processes is an important component of astrophysical studies. Despite this fact, in particular when it comes to the hot, diffuse, X-ray emitting intergalactic medium (IGM), where most baryons in the local Universe reside, current observational results largely yield only a static picture, as if reducing a more complex movie to a single freeze-frame. On these very large scales, it would take tens of millions to billions of years between observations to actually notice a change of structure, so dynamical information can only be obtained through measurements of the velocities in the IGM. The best way to perform these measurements directly is to obtain $\mathrm{X}$-ray spectra with a resolution better than at least a few $\mathrm{eV}$; the Hitomi satellite achieved a break-through in this field by providing spectra of this quality for the central region of the Perseus Cluster of galaxies. Upcoming $\mathrm{X}$-ray observatories promise to expand our detailed knowledge of the intracluster medium (ICM), the densest, brightest parts of the IGM, to a much larger sample of systems. In parallel, other observational methods based on X-ray imaging, optical or sub-millimetre spectroscopy, and the thermal and kinematic Sunyaev-Zel'dovich (SZ) effect have also been proposed as alternative tests for the strength of motions in the ICM, leading up to these future direct constraints from X-ray spectroscopy.

The goal of this review is to compile the existing quantitative constraints on the velocities of the intracluster plasma, and discuss their physical interpretation. We begin by summarising all the various processes that are expected to drive motions in the ICM from the perspective of theory and hydrodynamical simulations in Section 2, focusing on the expected magnitude, properties, and observational signatures specific to the velocity fields induced by cosmological large-scale structure formation (Section 2.1), idealised simulations of gas sloshing and ram-pressure stripped tails from subhalo infall (Sections 2.2 and 2.3, feedback from central active galactic nuclei (AGN; Section 2.4, and other effects such as plasma instabilities and motions of the member galaxies (Section 2.5). We then introduce various methods of determining the level of gas motions, and their results, in Section 3 . Section 4 discusses the interpretation of the inferred gas velocities in the context of identifying the source(s) of turbulence and constraining the microphysical properties of the ICM. Finally, in Section 5 we present the prospects of upcoming experiments that are expected to revolutionise this field of research.

In general, the current manuscript is not intended as a theoretical overview of the physics of turbulence in a magnetised plasma beyond the MHD view, for which we refer the reader to, e.g. Schekochihin and Cowley $(2006)$ for an introduction. There are many aspects of cosmological simulations of large-scale 

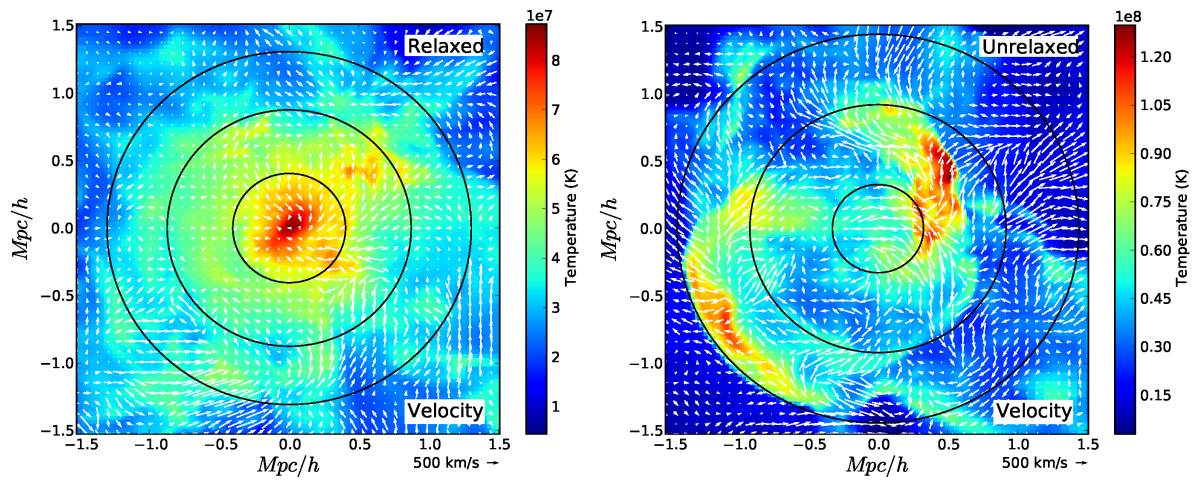

Fig. 1 Projected mass-weighted temperature map of a relaxed (left) cluster and an unrelaxed (right) cluster with the velocity vector fields overlaid from the Omega500 cosmological cluster simulation. The black circles denote $R_{2500}, R_{500}$ and $R_{200}$ of the clusters from inside to outside. Both the maps and vector fields are mass weighted along a $200 \mathrm{kpc} / \mathrm{h}$ deep slice centered on their respective cluster centers. Reproduced from Nelson et al. (2014a).

structure formation, the physics of gas sloshing, and AGN feedback that are not covered in this work; we have chosen to focus exclusively on how these processes affect the velocity structure of the ICM. Moreover, we only discuss the observational methods that provide independent constraints of the gas motions in individual systems, where the link to the dynamical state and disturbances in the gas density can be correlated in detail with the measured velocities to infer the physical processes that shape the kinematics of the ICM. The relevance of gas motions from the point of view of introducing deviations from the hydrostatic equilibrium (HSE) assumption and consequently influencing the measurement of cluster masses relevant for cosmological studies are addressed in the chapter by Pratt et al. in this volume.

\section{What are the physical processes that drive gas motions in the intergalactic medium?}

\subsection{Large-scale structure formation}

In the hierarchical structure formation model, clusters of galaxies form through a sequence of mergers and continuous mass accretion (Kravtsov and Borgani 2012). These merging and accretion events generate a significant level of gas motions inside the cluster potential well, which eventually heat the gas through shocks or turbulent dissipation (Figure 1). Hydrodynamical cosmological simulations of galaxy cluster formation using both grid-based (e.g., Norman and Bryan 1999, Ryu et al. 2003, Lau et al. 2009; Vazza et al. 2009, 2011; Iapichino et al. 2011; Nagai et al. |2013; Miniati 2014) and particle-based (e.g., Dolag et al. 2005: Battaglia et al. 2012) methods have found that the intracluster gas motions generated in the structure formation process are ubiquitous and contribute significantly to the energy and pressure budget throughout the ICM 
(Lau et al. 2009; Nelson et al. 2012, Zhuravleva et al. 2013a; Nelson et al. 2014b, Shi et al. 2015, 2018). The ratio of non-thermal pressure due to these gas motions with respect to the thermal pressure increases, on average, from $\sim 10 \%$ at $R_{500 c}$ to $\sim 30 \%$ at $R_{200 c}$ (Lau et al. 2009, Vazza et al. 2009; Battaglia et al. 2012), while the cluster core physics (such as radiative cooling and feedback from stars and AGN) drives additional gas motions in cluster cores (see Fig. 4 in Nagai et al. 2013, and 2.4 on the AGN feedback effect).

It is thought that gas motions are the dominant form of non-thermal pressure in galaxy clusters, with current radio and gamma-ray observations limiting the contribution from other sources (cosmic-rays and the magnetic field) at only a few percent of the thermal energy content in the virialized regions of these systems (e.g. Ackermann et al. 2010, Huber et al. 2013, Prokhorov and Churazov 2014: Ackermann et al. 2014 1 . Since X-ray and SZ observations typically measure only the thermal pressure component of the ICM, non-thermal pressure, if neglected, can introduce biases in the total energetics of the ICM as well as the hydrostatic mass estimation (e.g., Rasia et al. 2006, Nagai et al. 2007; Lau et al. 2009, Zhuravleva et al. 2013a; Biffi et al. 2016: Shi et al. 2016). For further detail, we refer the reader to the related chapter on "The galaxy cluster mass scale and its impact on cosmological constraints from the cluster population" (Pratt et al., submitted), in this topical collection.

\subsection{Cold fronts}

A particularly interesting manifestation of the ubiquitous motions associated with large-scale structure growth is represented by so-called "cold fronts". These sharp edges in X-ray surface brightness, where the brighter and denser side of the edge is colder than the other, were first discovered by Chandra Markevitch et al. 2000, Vikhlinin et al. 2001a) and are now known to be present in a large fraction of galaxy clusters (see the reviews by Markevitch and Vikhlinin 2007; Zuhone and Roediger 2016). Cold fronts occur in major mergers, such as in the "Bullet Cluster" (Markevitch et al. 2002) and Abell 3667 (Vikhlinin et al. 2001a), where the cold front is the contact discontinuity between the atmospheres of both merging subclusters. Cold fronts can also be produced by "sloshing" gas motions in the cool cores of clusters, triggered by perturbing subclusters, i.e., a minor merger (Markevitch et al. 2001, Ascasibar and Markevitch 2006). In this case the contact discontinuity is not between two parcels of gas initially belonging to different systems (as in the case of major mergers), but is due to gas from the cool core of a cluster being moved by the spiral-shaped sloshing motion out to a larger radius where it encounters ICM with a different entropy, temperature, metallicity, and density (e.g. Tanaka et al. 2006, Dupke et al. 2007b; Randall et al. 2009; Blanton et al. 2011). Idealised simulations of binary minor cluster mergers, tailored to specific sloshing

\footnotetext{
1 We note here that the gamma-ray and radio-based constraints might be weaker if the energy distribution of relativistic particles is softer than explicitly or implicitly assumed in these studies.
} 

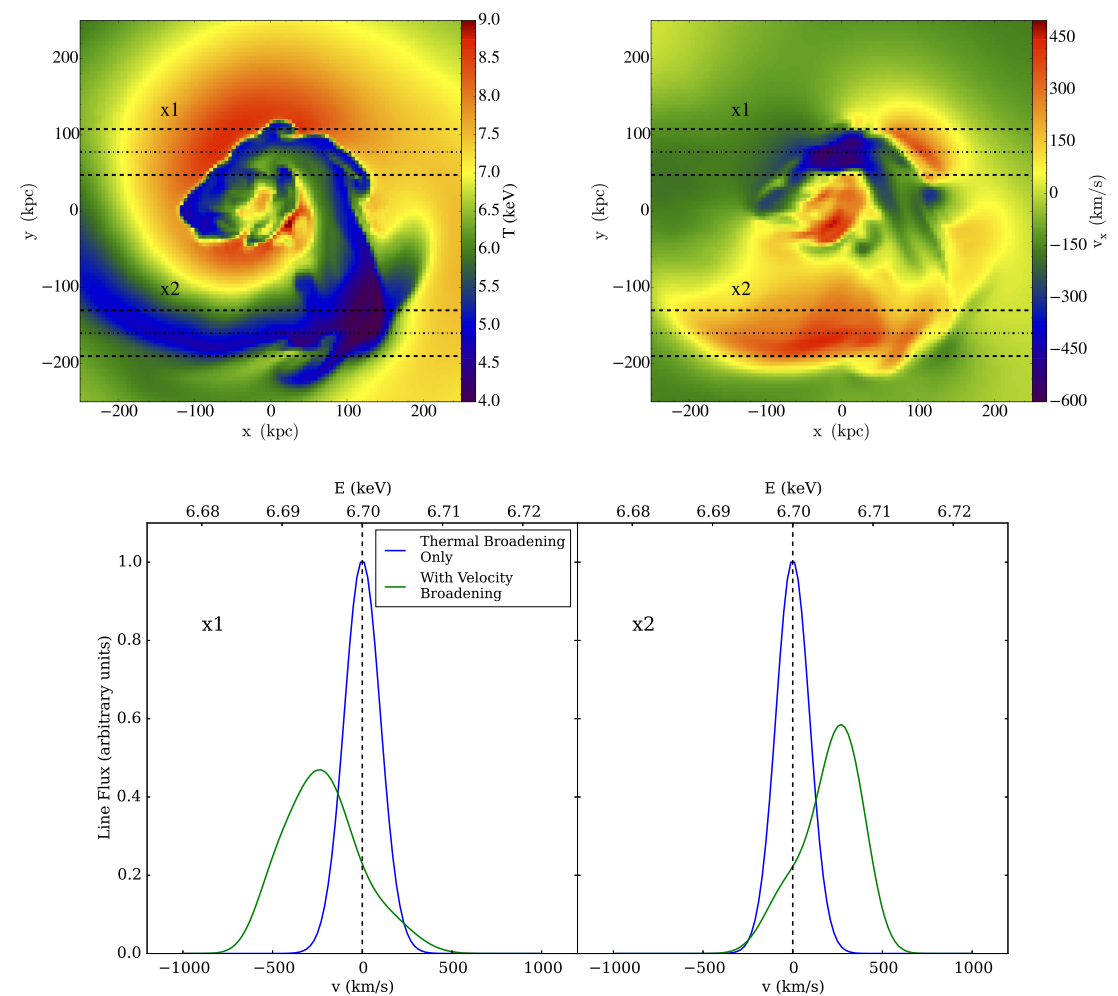

Fig. 2 Velocity fields of sloshing cold fronts from ZuHone et al. (2016). Top panels: slices of temperature (left) and velocity (right) with regions of interest marked by dashed and dotted lines. Bottom panels: shifting (mean velocity) and broadening (velocity dispersion) of a "toy" emission line which occurs due to the gas motions in the regions marked in the upper panels.

clusters in terms of the gravitational potential and ICM density and temperature profiles, can reproduce not only the locations of the observed sloshing cold fronts, but also the density and temperature contrasts across them (e.g., in Virgo and Abell 496, Roediger et al. 2011, 2012b). Thus, a careful interpretation of observed sloshing cold fronts, aided by tailored simulations, can be used to indirectly infer the current merger stage and the level of bulk motions. In addition, Zinger et al. (2018) showed that sloshing can be triggered also by penetrating gas streams arising from cosmic accretion. Though usually seen near the centers of clusters, a number of deep X-ray observations have even revealed indications of cold fronts at radii of up to $\sim 1 \mathrm{Mpc}$ (Simionescu et al. 2012, 2017; Paterno-Mahler et al. 2013; Rossetti et al. 2013, Walker et al. 2014, 2018a). In all cases, the fronts are contact discontinuities generated by subsonic gas motions. Numerical simulations suggest these motions have Mach numbers of $\mathcal{M} \sim 0.3-0.5$, corresponding to velocities of several hundred $\mathrm{km} \mathrm{s}^{-1}$. 
Cold fronts are thus observable indicators of bulk motions related to largescale structure growth and in general to all motions on spatially resolved scales in a medium with a substantial entropy gradient. Simulations have shown that characteristics of the observed velocity field depend strongly on the line of sight and its orientation with respect to the main direction of the cold front motion. For example, ZuHone et al. (2016) show that, at one extreme, if viewing the cluster along a line of sight perpendicular to the plane of its motion, both turbulence and oppositely directed large-scale bulk motions contribute mainly to an increase in the observed velocity dispersion $\left(\sim 100-200 \mathrm{~km} \mathrm{~s}^{-1}\right)$ with little effect on the mean velocity shifts of the ICM. With this orientation, the morphology of the surface brightness edge associated with the cold front also resembles most closely a spiral pattern. At the other extreme, if the cold front is viewed from a line of sight within or close to the plane of its motion, the oppositely directed parts of its overall motion will appear as a mean velocity gradient across a large region, in addition to significant line broadening that is again the result of both random turbulent motions and the smoothly varying bulk motion (see Figure 2). In this case, the spiral pattern in the surface brightness morphology disappears and is replaced by opposite and staggered, seemingly disconnected surface brightness edges (see also similar conclusions in Roediger et al. 2011). At intermediate viewing angles between these two extremes, both mean velocity gradients as well as velocity dispersions will be observed, along with a weaker spiral pattern in the density distribution. Combined constraints on the morphology and kinematics of cold fronts therefore provide a powerful tool promising to unlock details about the microphysical properties that govern the dynamics of the ICM.

\subsection{Subhalo infall and ram-pressure stripped tails}

Another obvious location to look for ICM motions and turbulence are the tails and wakes of subclusters or galaxies falling into their host clusters. Well-known examples include ESO 137-001 in Abell 3627 (Sun et al. 2010), elliptical galaxies in the Virgo cluster (M86, M89, M60, M49; described in, e.g., Ehlert et al. 2013; Kraft et al. 2017; Wood et al. 2017; Kraft et al. 2011), and the elliptical galaxy NGC 1404 in Fornax (e.g. Su et al.|2017a:|Sheardown et al.|2018). More recently, several apparently ram pressure stripped groups or galaxies with very long tails (several $100 \mathrm{kpc}$ ) have been discovered in the outskirts of massive clusters, e.g, in A2142, Hydra A or A85 (Eckert et al. 2014; Ichinohe et al. 2015; Eckert et al. 2017b; De Grandi et al. 2016). The previously known case of the NGC 4839 group in the Coma cluster (Neumann et al. 2001, Lyskova et al. 2018) belongs into the same category.

In the purely hydrodynamical case, the ICM flow patterns in and around such stripped subclusters or galaxies depend on the stage of the cluster crossing. The simplest case is the well-developed infall phase, where flow patterns of the ambient ICM closely follow the flow around a blunt body (Roediger et al. 2015a, see Fig 3). Along the upstream edge, the pressure follows the 

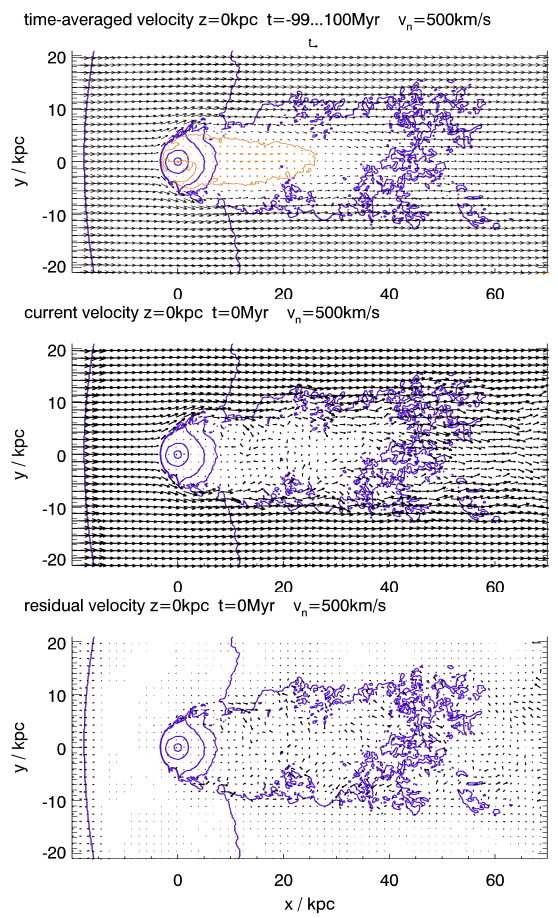

Fig. 3 Flow patterns around an elliptical galaxy falling into its host cluster, from Roediger et al. (2015a). The flow field is shown in the rest frame of the galaxy. The top, middle, and bottom panels show the time-average flow field, the current flow field, and the residual flow with respect to the averaged flow field, respectively. The purple contours show the gas density in a slice through the galaxy (logarithmic spacing, factor $\sqrt{10}$ ). The ICM is seen to flow around the remnant atmosphere similar to the flow around a blunt body. The downstream edge of the remnant atmosphere is at about the 4th innermost contour. Immediately downstream of the remnant atmosphere is the deadwater region, where on average the flow is directed back to the galaxy, i.e., into the upstream direction, as indicated by the red contour and the red flow vectors in the top panel. The deadwater region as well as the further downstream wake are turbulent (in the absence of mechanisms suppressing turbulence) for many atmosphere diameters downstream of the galaxy.

well-known distribution of an enhanced pressure at the upstream stagnation point, decreasing towards the sides of the stripped atmosphere. Observational data now have reached a quality where these pressure gradients along the upstream edge can be measured and used to infer the 3D motion of the subcluster, as done by Su et al. (2017a) for NGC 1404 in Fornax. Downstream of the stripped atmosphere, the flow is characterised by a downstream deadwater region and a turbulent wake.

If the ICM can indeed be modelled largely by pure hydrodynamics, such wakes are potentially very long-lived, similar to the wake of a bullet in air shown in van Dyke (1982). A notable difference between the flow past a blunt body and a gas-stripped subcluster is the fact that the shape of the "body" in 
the flow changes. Specifically, the subcluster's gas is stripped predominantly in the upstream region and along its side, but the downstream atmosphere is shielded from the ICM head wind and can persist as a bright tail even after the subcluster passes the core of the main cluster. At this stage, the ICM flow patterns become more complex, as the subcluster moves towards the apocentre of its orbit. The rapid decrease of ram pressure experienced by the subcluster leads to its tail falling back towards the subcluster potential and overshooting the potential centre in a slingshot fashion. Likely several of the very long tails of subclusters in the main cluster outskirts are such slingshot tails and not simple ram pressure tails (as proposed, for example, for Abell 168, Hallman and Markevitch 2004, and the Pandora Cluster, Owers et al. 2011; Merten et al. 2011). While the subcluster is lingering near its apocentre, the ICM flow patterns are far from the regular blunt-body-in-a-flow, and observations need to be interpreted carefully (for details see Sheardown et al, in submitted). Beyond the pure hydrodynamic view, Vijayaraghavan and Sarazin (2017b a ) show that unsuppressed thermal conduction, even saturated, would erase the tails of stripped ellipticals, while anisotropic suppression of thermal conduction due to magnetic fields allows the tails to survive.

\subsection{AGN feedback}

While mergers are certainly the main source of gas motions in the bulk of the ICM, in the cores of relaxed clusters the energy input from the central supermassive black holes is plausibly the dominant mechanism that perturbs the gas. Here, the ICM radiative cooling time $t_{c o o l}$ is typically an order of magnitude (or more) shorter than the Hubble time, implying that without an external source of energy the gas should flow from radius $r$ towards the center of the potential well with the velocity $v \sim \frac{r}{t_{c o o l}}$ (see Fabian 1994, for a review of the cooling flow paradigm) and accumulate there in the form of stars or cold gas. However, such a concept contradicts many observational constraints (for a review, see Peterson and Fabian 2006), suggesting that there must be a source of energy that compensates for the ICM radiative losses.

It is well established that most galaxies harbor a central SMBH with masses ranging between $10^{6}-10^{10} M_{\odot}$ (e.g., Kormendy and Ho 2013 for a review). The amount of energy released by such black holes is sufficient to expel the gas from galaxy-size halos (e.g. Silk and Rees 1998) or compensate gas cooling losses in more massive systems (e.g. Ciotti and Ostriker 2001, see however Fujita and Reiprich 2004). Indeed, the total feedback energy related to such SMBHs is $\varepsilon_{\mathrm{m}} M_{\mathrm{BH}} c^{2} \approx 10^{58}-10^{62}$ erg (with a mechanical efficiency $\varepsilon_{\mathrm{m}}=3 \%$; Sądowski and Gaspari 2017). Early X-ray and radio observations revealed that in many cool-core clusters there are bubbles of radio-bright plasma (e.g. Böhringer et al. 1993) associated with the central SMBHs, which apparently are able to inflate cavities (X-ray dim regions). Such cavities are expected to be buoyant and must rise in the stratified atmospheres (Gull and Northover 1973). The comparison of the inflation and buoyancy time scales for the cav- 
ities led to the conclusion that the characteristic mechanical powers of the SMBHs in the Perseus (Churazov et al. 2000) and Hydra A (McNamara et al. 2000 ) clusters are comparable with the ICM cooling losses. This conclusion has been confirmed with Chandra and XMM-Newton observations for a large sample of clusters and groups spanning a wide range of masses and luminosities (e.g. Bîrzan et al. 2004, Hlavacek-Larrondo et al. 2012, see Fabian (2012); McNamara and Nulsen (2012) for reviews), suggesting that feedback from AGNs can prevent catastrophic cooling of the gas. Here we focus on the gas velocities induced by AGN feedback in conditions relevant for galaxy clusters, rather than for lower mass systems, where different aspects of AGN-gas interactions might be important.

By now, there is a rich landscape of models that consider different flavors of the AGN feedback (see, e.g., the related chapter in this book, Werner et al. 2019). While there is a general agreement on the importance of AGN feedback for the energy balance in cluster cores, the uncertainties in the form of the energy release by the AGN and in the physical mechanisms that lead to the energy dissipation are still very large. Measuring gas velocities can eliminate some of these uncertainties.

The first group of models assumes that bubbles are filled with relativistic particles or very hot plasma, which remain confined within the bubbles, implying that there is no direct heat exchange with the ICM. In these models, the energy goes from the bubbles to the ICM via, e.g., the generation of sound or internal waves, turbulence in the wake of the bubbles, and entrainment of the low entropy gas, which all have different signatures in the observed gas velocities.

A powerful injection of energy into an unperturbed hydrostatic atmosphere is naturally accompanied by shocks (e.g. Heinz et al. 1998, Reynolds et al. 2002). This is true for spherical bubbles during their initial (fast) expansion phase and even more important for collimated outflows with large momenta. Many examples of weak shocks/sound waves are found in cluster cores (e.g. Fabian et al. 2003a; McNamara et al. 2005, Forman et al. 2007, 2017, Randall et al. 2015). This led to the suggestion that most of the AGN energy goes into the generation of shocks and sound waves, which propagate radially and eventually dissipate due to the viscosity and conductivity of the ICM (e.g. Fabian et al. 2003a, 2006, 2017, Ruszkowski et al. 2004, Gaspari et al. 2011. Barai et al. 2016; Li et al. 2017). Other studies argue that the fraction of energy that is carried by sound waves is subdominant (e.g. Churazov et al. 2000, Zhuravleva et al. 2016; Forman et al. 2017; Tang and Churazov 2017) since, for a very fast expansion velocity, the energy is dissipated locally by a strong shock, while for a slow expansion the generation of sound waves is inherently inefficient. Nevertheless, if the outflow is collimated and has significant momentum, so that the head of the jet is always moving trans-sonically, sound waves can still play an important role. In terms of gas velocities, the sound waves scenario predicts quasi-spherical and almost radial motions (at some distance from the cluster center). The wave amplitudes decrease with radius due to the spherical geometrical factor and due to dissipation. In this scenario, 
the observed X-ray lines would be very broad and have boxy shapes towards the center (for spatially resolved wave patterns, but might be peaked for unresolved ones) and become progressively narrower with the projected distance from the center (e.g. Rebusco et al. 2008; Heinz et al. 2010, Zhuravleva et al. 2011).

A quasi-continuous energy injection scenario argues instead that the dominant fraction of energy goes into the enthalpy of the bubbles (Churazov et al. 2000). While there is a generic energy conservation argument stating that the buoyantly rising bubbles transfer most of their energy to the gas after crossing several pressure scale heights of the atmosphere (Churazov et al. 2001, Begelman 2001, Churazov et al. 2002), this argument does not specify how the energy is extracted from the bubbles and how this energy is dissipated. Depending on the properties of the ICM (e.g., viscosity) and characteristics of the bubbles, the energy can be converted, for instance, into turbulence in the wake of the bubble or into internal waves (e.g. Churazov et al. 2000, Omma and Binney 2004 Gaspari et al. 2012b; Zhang et al. 2018). The latter scenario is attractive since the internal waves are trapped in cluster cores (Balbus and Soker 1990), but can spread the energy in the tangential direction. As with sound waves, several other studies (e.g. Reynolds et al. 2015, Bambic et al. 2018) argue that internal waves are not playing an important role in the energy flows in cluster cores (see, e.g., the mixing scenarios below), or that the waves do not propagate fast enough in the vertical direction. The latter problem is alleviated by noting that the radial energy transport is provided by the bubbles themselves rather than by the waves. In terms of the velocity field characteristics, the main difference with the sound waves scenario is the predominance of the tangential over radial velocities. In contrast to radial sound waves, the lines could be narrower towards the cluster center but become broader at larger radii (e.g. Rebusco et al. 2008; Zhuravleva et al. 2011). This conclusion may also depend on whether the spatial pattern is resolved or whether one observes the line from the entire region within a given radius. If the bubbles rise fast enough, the excitation of internal waves is inefficient and a hydrodynamic drag related to the turbulence in the wake of the bubble may dominate.

The energy (enthalpy) of the bubbles can also go into entrainment of the low entropy gas from the central region to larger radii. Examples of structures that are likely formed by such entrainment are found in X-ray observations of many clusters (e.g. Simionescu et al. 2008, 2009, Gitti et al. 2011; Kirkpatrick et al. 2009, 2011; Kirkpatrick and McNamara 2015). Thermal instabilities are likely to develop in such entrained flows (as pointed out by Churazov et al. 2001). Indeed, cool core clusters are frequently observed to host filamentary nebulae of multi-phase gas with temperatures ranging from $<100 \quad 10^{4} \mathrm{~K}$ (see Section 3.1.7).

The formation and evolution of these filaments has been studied in detail through a suite of numerical simulations that include the effect of radiative cooling. The cooling instability has been discussed intensively in the cooling flow models (e.g. Fabian and Nulsen 1977; Mathews and Bregman 1978), 

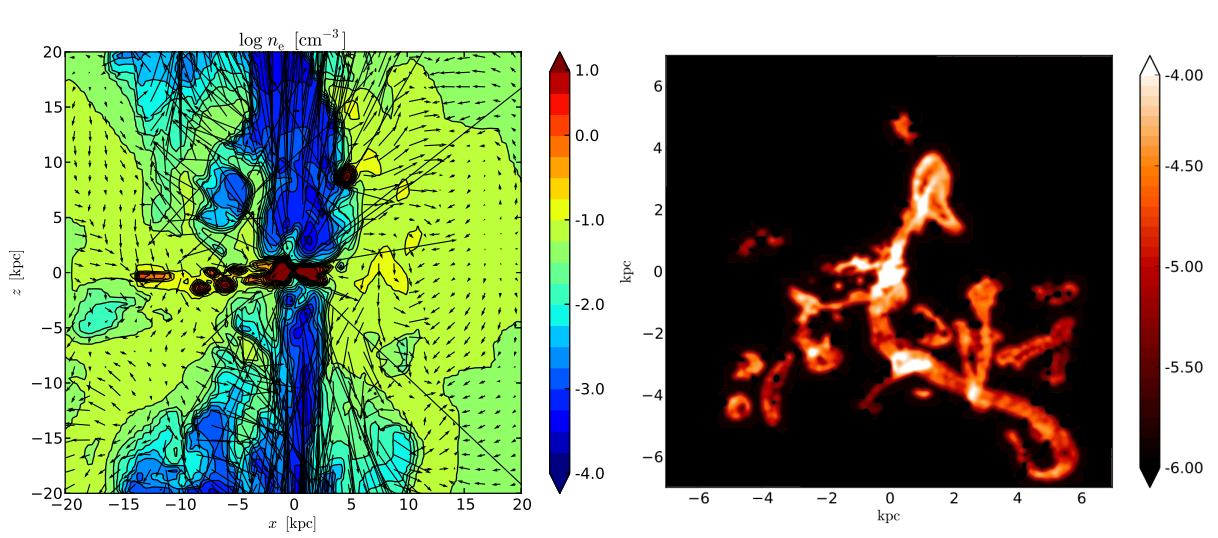

Fig. 4 The tight relation between turbulence and AGN feedback and feeding properties in X-ray halos. Left: electron number density cross-section of a BCG, showing an ultrafast outflow/jet triggered by a previous CCA rain phase (clouds in red) which generates large-scale cavities, shocks, and chaotic motions (velocity field overlaid as black arrows); reproduced from Gaspari et al. (2013b). Right: surface brightness map of the condensed gas - turbulence-driven density fluctuations have become nonlinear, leading to a top-down multiphase condensation phase of warm $\left(\sim 10^{4} \mathrm{~K}\right)$ filaments and clouds out of the diffuse hot plasma, which will soon boost the SMBH accretion rate via CCA and trigger a new generation of AGN jets/outflows; reproduced from Gaspari et al. (2017).

although Malagoli et al. (1987); Balbus and Soker (1989) have shown that linear perturbations do not grow. Pizzolato and Soker (2005) suggested that non-linear density perturbations, possibly seeded by an earlier radio jet, would cool to low temperatures $\left(\mathrm{T}<10^{4} \mathrm{~K}\right)$ and feed the black hole. In terms of the evolution of density perturbations evolving from either linearly idealized (McCourt et al. 2012; Sharma et al.|2012) or realistic AGN jet conditions (Gaspari et al. 2012a. 2013b, see left panel in Fig. 4), it has been suggested that thermal instabilities can in fact develop throughout the volume of the cool core when the ratio of the cooling time and free-fall time, $t_{\text {cool }} / t_{f f}$, falls below roughly a factor of 10. Numerical simulations went on to demonstrate that uplift of lowentropy ambient gas along the direction of the rising radio bubbles is at least as important to the production of cold clouds (Revaz et al. 2008; Li and Bryan 2014). The conditions under which thermal instabilities develop are discussed in more detail in the chapter on "Hot X-ray Atmospheres, Molecular Gas and AGN Feedback in Early Type Galaxies: A Topical Review", in this volume (Werner et al. 2019). In any case, it is generally clear that the combination of gas motions triggered by the AGN (either in the form of volume-filling turbulence or directed entrained outflows), together with a short central cooling time and consequently a low $t_{c o o l} / t_{f f}$, correlate with and are likely responsible for the onset of cooling of the X-ray emitting ICM and the formation of optical and sub-millimetre line emitting filaments.

The presence of thermal instabilities and cold(er) gas phases embedded in the ICM is thought to be crucial for regulating the AGN feeding and feedback cycle (Figure 4 , right panel). The AGN jet feedback typically drives a turbu- 
lent velocity dispersion $\sigma_{v} \approx 100-300 \mathrm{kms}^{-1}$ (as shown by high-resolution hydrodynamical simulations in Gaspari et al. 2012a b, 2018, Valentini and Brighenti 2015), which enables both a self-consistent gentle circulation and the seeding of nonlinear (lognormal) gas fluctuations (see also Fig. 6). In analogy to Earth weather, warm extended filaments and cold clouds condense out of the hot ICM and rain toward the central SMBH (Gaspari et al. 2012a 2015, 2017, Voit et al. 2015, Voit 2018, Prasad et al. 2017). Inelastic collisions in the central kpc region promote angular momentum cancellation and boost the AGN feeding rate by 100 fold, in a process known as Chaotic Cold Accretion (CCA; Gaspari et al. 2013a; see also Sec. 3.1.7). The accretion of cold gas triggers a new generation of AGN feedback injection. The resulting subsonic turbulence mixes the gas in an eddy turnover time, $t_{\text {eddy }}=L / \sigma_{v}$, where $\mathrm{L}$ is the injection scale of the size of the bubble diameter. Later, as the cooling time lowers back again and $t_{\text {cool }} / t_{\text {eddy }} \approx 1$, a new cycle of CCA rain and selfregulated AGN feedback restarts (Gaspari and Sądowski 2017 for a review of the self-regulation). This duty cycle continues for several billion years and has to preserve the cool-core structure since at least redshift $z \sim 2$ (McDonald et al. 2017).

In addition, there are also many more numerical simulations that include other physical mechanisms, e.g., magnetic field effects (see Vernaleo and Reynolds 2006: Ruszkowski et al. 2017b; Bambic et al. 2018), explicit viscosity (Reynolds et al. 2015), or streaming of cosmic rays (Boehringer and Morfill 1988: Loewenstein et al. 1991; Wiener et al. 2017, Ruszkowski et al. 2017a), whose distinct features are also partly reflected in the gas velocity fields. The vast majority of models mentioned in this section predict mostly subsonic velocities in the bulk of the ICM within the cluster cooling radius ${ }^{2}$. This is a rather natural result, given that a) strongly supersonic motions decay very quickly and are therefore short-lived and b) the large ratio between the cooling time and the free fall time implies that at least in a quasi-steady scenario supersonic motions in the bulk of the ICM are not required and would instead overheat the gas. Given the superposition between the motions due to AGN feedback and those driven by mergers on larger scales (as discussed in the previous subsections), the question arises whether the predictions by the large range of models described above are different enough to allow an easy differentiation between various scenarios.

In a different group of models, the AGN injects hot (but not relativistic) plasma ${ }^{3}$ that can mix with the ICM (Brüggen and Kaiser 2002; Pizzolato and Soker 2005; Reynolds et al. 2015). Such mixing is naturally present in numerical simulations that use grid-based schemes, but might be suppressed in SPHbased codes (Sijacki and Springel 2006). At low resolution, the numerical mixing might be relevant; however, in modern simulations with intermediate/high

\footnotetext{
2 These arguments do not exclude though a possibility of having localized regions with supersonic motions, especially in the regions where the AGN-driven outflows/jets interact with the ICM (e.g. Heinz et al. 2010).

3 Such plasma could also appear as the result of ICM heating by strong shocks, if the energy is released by an AGN in the form of short and powerful outbursts.
} 
resolution, the mixing is dominated by physical turbulent mixing and hydrodynamical instabilities (such as Rayleigh-Taylor or Kelvin-Helmholtz). Mixing is a very efficient process of sharing the injected energy with large volumes of the ICM and other heating channels might be subdominant. CARMA maps show a deficit in the SZ signal coincident with the X-ray identified cavities in MS 0735.6+7421 (Abdulla et al. 2018), suggesting that they are unlikely to be supported thermally, although extremely diffuse thermal plasma with temperatures in excess of hundreds of $\mathrm{keV}$ cannot be ruled out as the main component of the bubbles. If the injected plasma is very hot, but not relativistic, then the most important constraints on AGN heating might come not from the gas velocities but from the broad temperature distribution of the mixing plasmas.

\subsection{Other sources of turbulence in the intra-cluster medium}

In addition to the structure formation process and AGN feedback, cluster member galaxies also influence the dynamics of the surrounding ICM, even in relaxed systems. Ruszkowski and Oh (2011) show that galaxies moving through the host cluster excite large-scale g-modes, leading to volume filling turbulence with a velocity dispersion in the central $100 \mathrm{kpc}$ estimated at $150-200 \mathrm{~km} / \mathrm{s}$.

The level of gas motions in the ICM can also be affected by instabilities inherent to a stratified low-collisionality plasma in the presence of a weak magnetic field. If anisotropic thermal conduction is the dominant mode of heat transport, instabilities can develop on macroscopic scales. Examples include the magnetothermal instability (MTI) (Balbus 2000: Parrish and Stone 2005), which is important in the case of a negative temperature gradient usually found in the cluster outskirts (Parrish et al. 2012), and the heat buoyancy instability (HBI) (Quataert 2008), which occurs for a positive temperature gradient (for instance in the cores of relaxed clusters, where the temperature increases as a function of radius). However, Ruszkowski and Oh (2010) argued that even a very low level of turbulent perturbations, entirely consistent with the expectations for cosmological infall, galaxy motions, mergers, or AGN activity, can entirely alter the magnetic field distribution resulting at least from the HBI instability, preventing it from saturating.

Furthermore, any turbulent stresses and the resulting local changes in the magnetic field will trigger very fast micro-scale instabilities, such as the firehose, mirror, and gyrothermal instability (e.g. Schekochihin et al. 2005, 2010 Lyutikov 2007). These instabilities affect the large-scale transport properties of the ICM, including its effective viscosity and consequently its dynamics. Kunz et al. (2011) propose that parallel viscous heating, due to the anisotropic damping of turbulent motions, is regulated by the saturation of micro-scale plasma instabilities; if this is assumed to be the source of heating that balances radiative cooling in the cores of galaxy clusters, the predicted ICM turbulent velocities are of order $100-200 \mathrm{~km} / \mathrm{s}$ for an A1835-like cluster. 


\section{Observational probes of the ICM velocity}

3.1 State of the art in the absence of high-resolution, nondispersive X-ray spectrometers

\subsubsection{Line shifts}

The simplest manifestation of bulk motions in the ICM is the Doppler shift of X-ray spectral emission lines. The magnitude of the energy shift is approximately given by $\Delta E=E_{\text {line }} \times v_{\text {bulk }} / c$, where $E_{\text {line }}$ is the expected position of the spectral line in the absence of gas motions, $v_{\text {bulk }}$ is the line-of-sight velocity of the gas (note that motions in the plane of the sky cannot be detected in this way), and $c$ is the speed of light. Hence, these measurements are extremely challenging, since even a $1 \%$ uncertainty in calibrating the detector gain corresponds to an error of $3000 \mathrm{~km} / \mathrm{s}$ in the bulk velocity. Initial attempts to constrain the magnitude of gas motions using ASCA and later Chandra and $X M M-N e w t o n$ data had suggested large line-of-sight velocity gradients of order a few thousand $\mathrm{km} / \mathrm{s}$ in the Perseus and Centaurus clusters (Dupke and Bregman 2001ba, 2006), as well as the merging cluster Abell 576 (Dupke et al. 2007a), although the associated uncertainties were themselves of order $1000 \mathrm{~km} / \mathrm{s}$. Using the ${ }^{55} \mathrm{Fe}$ calibration source onboard the Suzaku satellite, the absolute energy scale calibration of the XIS detectors can be determined more accurately (with a precision as good as 0.1\%, Ozawa et al. 2009), allowing somewhat tighter constraints to be placed on the spectral line shifts from several galaxy clusters; at 90\% confidence, Ota et al. (2007) place an upper limit of $1400 \mathrm{~km} / \mathrm{s}$ line-of-sight velocity gradient in the Centaurus Cluster, and Sugawara et al. (2009) find that the bulk motions in Abell 2319 do not exceed $2000 \mathrm{~km} / \mathrm{s}$.

In addition to these upper limits, several detections of gas bulk motions were reported from Suzaku data. In the merging cluster Abell 2256, Tamura et al. (2011) found a significant line of sight velocity gradient of $1500 \pm 300$ (statistical) \pm 300 (systematic) km/s. In the Perseus Cluster, Tamura et al. (2014) discovered a hint of gas bulk motions at the level of only $-(150-300) \mathrm{km} / \mathrm{s}$ relative to NGC1275; this velocity gradient is spatially coincident with the cold-front located 45-90 kpc west of the cluster center. Ota and Yoshida (2016) performed a search for line shifts with respect to the expected rest frame in a sample of nearby clusters of galaxies with various X-ray morphologies observed with Suzaku; they report upper limits of $3000-4000 \mathrm{~km} / \mathrm{s}$ in A 2199, A 2142, A 3667, and A 133, and hints of large bulk velocities in excess of the instrumental calibration uncertainty near the center of the cool-core cluster A2029 and in the subcluster of the merging cluster A2255.

In summary, X-ray CCD detectors typically indicate that gas bulk motions in cluster centers are below a few thousand $\mathrm{km} / \mathrm{s}$, but significant detections of line shifts remain elusive - particularly in relaxed systems. 


\subsubsection{Line broadening}

In addition to the centroids of X-ray spectral emission lines, their widths also hold important clues regarding the gas dynamics. Thermal motions of the respective ion, turbulent gas motions along the line of sight, and the response of the instrument all contribute in quadrature to the observed width of a line: $W_{\mathrm{obs}}^{2}=W_{\text {therm }}^{2}+W_{\text {turb }}^{2}+W_{\text {inst }}^{2}$. The thermal motions can be calculated as

$$
W_{\text {therm }}=\frac{\nu_{0}}{c} \sqrt{\frac{k_{B} T}{A m_{p}}}
$$

where $k_{B}$ is the Boltzmann constant, $m_{p}$ the proton mass, $A$ the atomic weight of the element, and $\nu_{0}$ the frequency of the transition. The turbulent line broadening meanwhile is written as

$$
W_{\mathrm{turb}}=\frac{\nu_{0}}{c} \sigma_{v \|}
$$

where $\sigma_{v \|}$ is the line of sight component of the turbulent velocity. For the case of isotropic turbulence, $\sigma_{v \|}^{2}=\sigma_{v}^{2} / 3$, where $\sigma_{v}$ is the root mean square of the three-dimensional turbulent velocity. Because only $\sigma_{v \|}$ affects the observed spectral line widths, throughout this paper we define the observed turbulent velocities $v_{\text {turb }} \equiv \sigma_{v \|}$. It should be noted that both $W_{\text {therm }}$ and $W_{\text {turb }}$ are typically of order a few eV; hence, CCD spectrometers with $W_{\text {inst }}>100$ $\mathrm{eV}$ cannot be used to constrain the level of turbulent motions in the ICM because the instrumental line broadening dominates over the expected Doppler broadening.

The first successful attempts to constrain the level of turbulence in galaxy cluster cores from X-ray line widths were instead performed using the Reflection Grating Spectrometers (RGS) onboard XMM-Newton. The RGS has a remarkable energy resolution of only $\sim 2-3 \mathrm{eV}$ at $1 \mathrm{keV}$ for a point-like source. However, its nature as a slit-less spectrometer complicates the analysis of extended objects such as diffuse, nearby clusters of galaxies. This is because photons originating from a region that is offset in the direction along the dispersion axis are slightly shifted in wavelength, resulting in changes in the line position and width. This effect can be corrected for, approximately, by using a surface brightness profile extracted along the dispersion direction and convolving it with the instrumental line shape. Note, however, that the spatial distribution of various ions $(\mathrm{O}, \mathrm{Fe}$, etc) may not be exactly the same as that of the average gas density (for example, due to a radial gradient of the ICM metallicity), making this approach somewhat uncertain. It is clear that the correction to the line width is smallest (and hence uncertainties in this correction have the least effect on the measurements) for clusters with a very compact, sharp central X-ray emission peak. Taking advantage of this strategy, Sanders et al. (2010a) placed the first direct limit on the turbulent velocity in Abell 1835 of $v_{\text {turb }}<274 \mathrm{~km} / \mathrm{s}$ (at 90\% confidence), while Bulbul et al. (2012) report an upper limit of $v_{\text {turb }}<206 \mathrm{~km} / \mathrm{s}$ in Abell 3112. Sanders and Fabian 
(2013) and Pinto et al. (2015) expanded this method to larger samples of clusters, groups of galaxies, and elliptical galaxies. The results by Pinto et al. (2015) were based on the CHEmical Enrichment RGS Sample (CHEERS), designed as a "complete" sample of high-quality RGS cluster spectra within $z<0.1$ that can be obtained within a reasonable exposure time of less than $200 \mathrm{ks}$ each (de Plaa et al. 2017). This study found that one half of the 44 objects in the sample had turbulent velocities $v_{\text {turb }} \equiv \sigma_{v \|}$ below $350 \mathrm{~km} / \mathrm{s}$ (at $90 \%$ confidence), while in about a quarter of the objects values as high as $v_{\text {turb }}>700 \mathrm{~km} / \mathrm{s}$ were still allowed by the data.

\subsubsection{Resonant scattering}

Although the ICM is generally optically thin, if the gas turbulent velocities are negligible compared to the thermal velocities of ions, then several of the brightest X-ray emission lines are expected to become moderately optically thick in the cores of galaxy clusters, groups and massive elliptical galaxies. Because the optical depth is highest in the central region of a cluster, a fraction of the photons originating from that region are expected to be resonantly scattered away from the line-of-sight, causing an apparent suppression of the resonant line relative to other lines (Gilfanov et al. 1987). Since the optical depth is sensitive to the turbulent broadening, one can probe gas velocities in the hot haloes of clusters and galaxies by measuring the apparent flux suppression, even if the lines are not fully resolved spectrally. We refer the reader to the chapter on "X-ray spectroscopy of galaxy clusters: beyond the CIE modeling" in this volume (Gu et al. 2018) for a more detailed discussion on the effects of resonant scattering on the X-ray spectra of the ICM; here, we focus solely on the turbulent velocity constraints obtained from this method.

Early XMM-Newton CCD spectrometer data did not reveal a strong suppression of the resonant He-like Fe line at $6.7 \mathrm{keV}$ in the core of the Perseus Cluster (Churazov et al. 2004, Gastaldello and Molendi 2004), which at face value would imply that strong turbulent motions are present in this region. It was later shown that, with the limited energy resolution of CCDs, it is difficult to cleanly single out the magnitude of resonant scattering when variations of gas temperature and metallicity are present (Zhuravleva et al. 2013b). This difficulty also explains some controversial results obtained for other clusters (e.g. Kaastra et al. 1999, Akimoto et al. 2000, Mathews et al. 2001, Sakelliou et al. 2002; Sanders and Fabian |2006).

Clear evidence of the resonant scattering effect was found using highresolution XMM-Newton RGS spectra of the cooler gas in the cores of giant elliptical galaxies and groups. A strong suppression of the Ne-like Fe line at $15.01 \AA$ was detected in many galaxies, including NGC4636, NGC1404, NGC5813 and NGC4472 (Xu et al. 2002, Kahn et al. 2003; Werner et al. 2009 de Plaa et al. 2012). Werner et al. (2009) and de Plaa et al. (2012) modeled the resonant scattering effect for different values of the characteristic turbulent velocities and, by comparing the data to the models, found $v_{\text {turb }}<100 \mathrm{~km} / \mathrm{s}$ in the inner kpc region in NGC 4636, $320<v_{\text {turb }}<720 \mathrm{~km} / \mathrm{s}$ in NGC5044 

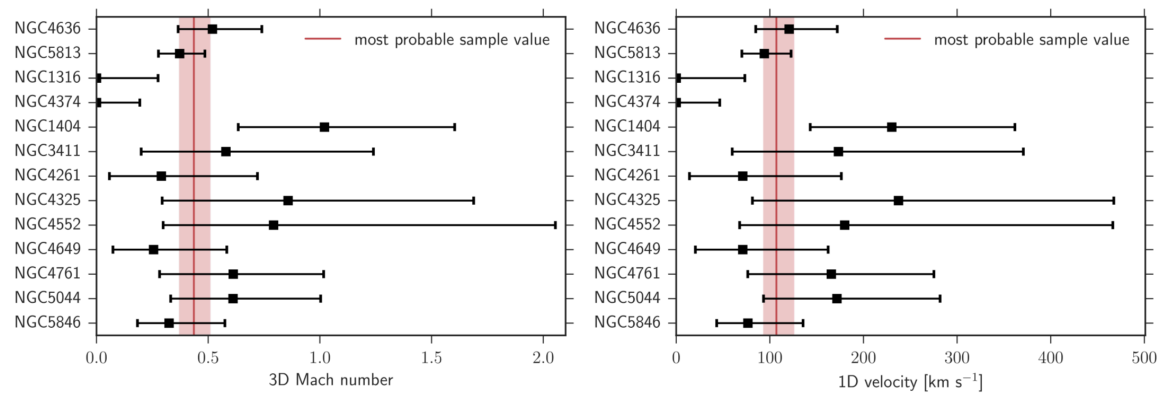

Fig. 5 Combined resonant scattering and line broadening constraints on 3D Mach number (left) and 1D turbulent velocity amplitude (right) for 13 galaxies with $1 \sigma$ uncertainties. Red lines and regions show best fit most probable sample values. Adapted from Ogorzalek et al. (2017).

and $140<v_{\text {turb }}<540 \mathrm{~km} / \mathrm{s}$ in NGC5813. More recently, Ogorzalek et al. (2017) extended the resonant scattering analysis to a sample of 13 elliptical galaxies, finding velocities ranging between $0-120 \mathrm{~km} / \mathrm{s}$ in the cores of four massive galaxies and placing lower limits of $20-100 \mathrm{~km} / \mathrm{s}$ in the remaining nine objects. Combining the line broadening results of Pinto et al. (2015) and the resonant scattering results, Ogorzalek et al. (2017) obtained velocity constraints for all 13 galaxies, with the sample best-fit mean value of $v_{\text {turb }} \sim 110$ $\mathrm{km} / \mathrm{s}, 3 \mathrm{D}$ Mach number of $\sim 0.45$, and non-thermal pressure fraction $\sim 6$ per cent (Fig. 5 ).

\subsubsection{Surface brightness fluctuations and power spectra}

The idea behind the gas velocity measurements from surface brightness fluctuations is simple: any deviations of the hot gas from hydrostatic equilibrium should be associated with gas motions leading to perturbations in X-ray images relative to a smooth, undisturbed model. Various physical mechanisms behind this correlation as well as different approaches to extract this information from observational data have been discussed in Schuecker et al. (2004); Churazov et al. (2012); Sanders and Fabian (2012); Gaspari and Churazov (2013); Gaspari et al. (2014); Zhuravleva et al. (2014a b). The study of Schuecker et al. (2004) was focused on the case of isotropic turbulence in a homogeneous fluid that leads to pressure fluctuations scaling as the square of gas velocities. In Churazov et al. (2012) several other processes contributing to X-ray surface brightness fluctuations have been added, including perturbations of gravitational potential, entropy and density perturbations due to turbulent motions in stratified atmospheres, density variations in sound waves, metallicity variations and bubbles of relativistic plasma.

By using high-resolution 3D hydrodynamic simulations probing varying levels of turbulence in the electron-ion ICM, Gaspari and Churazov (2013) first showed that the normalization (amplitude $A_{\delta}$ ) of the X-ray density power spectrum is linearly related to the turbulent Mach number (unlike the above 

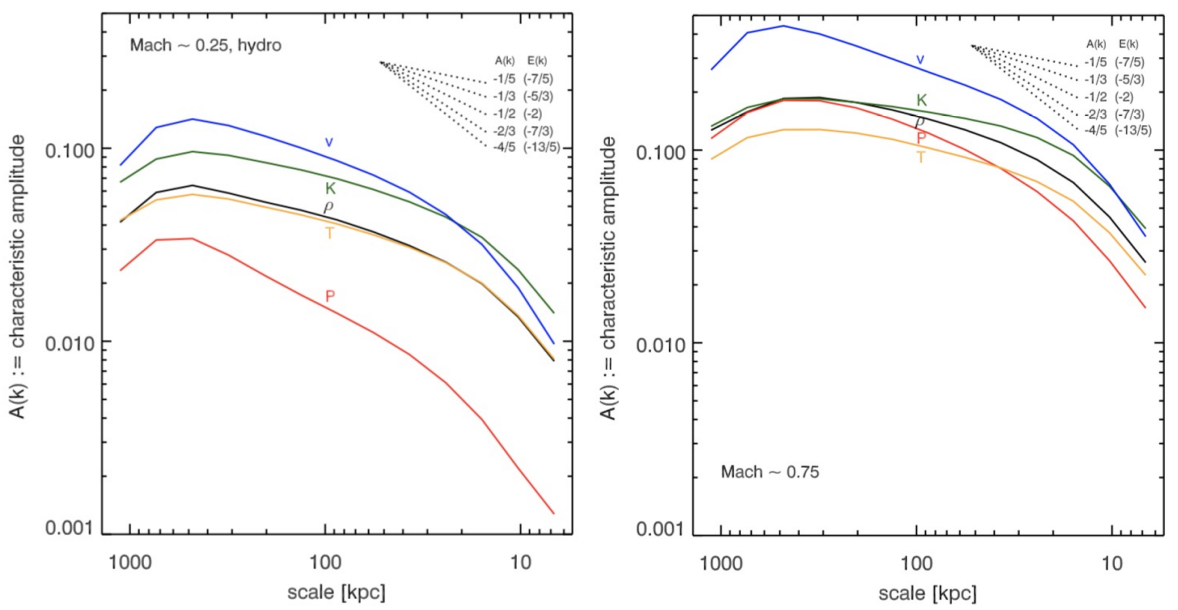

Fig. 6 Power spectra of all thermodynamic fluctuations (density, temperature, pressure, entropy) from high-resolution hydrodynamical simulations of turbulence in a stratified Comalike cluster (no conduction). Left: Mach 0.25 (weak) turbulence. Right: Mach 0.75 (strong) turbulence. It is clear that the amplitude of thermodynamic fluctuations is linearly related to the turbulent Mach number, while the slope depends on the specific transport properties of the gas (and does not necessarily behave as a passive scalar). Reproduced from Gaspari et al. (2014).

suggestions of a square dependence): $A_{\delta} \simeq 0.25 \mathrm{Mach}_{3 \mathrm{D}}$. The same suite of simulations showed that it is possible to convert the density spectrum into a velocity spectrum (Fig. 6), although below the injection scale, the linear relation tends to progressively loosen up, in particular in the presence of strong thermal conduction. The steepening of the slope (from Kolmogorov toward Burgers) represents another way to probe the plasma microphysics in the ICM. Gaspari et al. (2014) further showed the power spectra evolution of all the thermodynamic quantities: for $M<0.5$ the entropy power spectrum (internal waves) dominates over that of pressure (sound waves), while for $M>0.5$ pressure waves start to become substantial. Increasing levels of turbulent motions thus move the ICM from the isobaric to the more compressive, adiabatic regime (Fig. 6).

When all perturbations in a stably stratified atmosphere are infinitesimal and all motions are slow $(\mathrm{M} \ll 1)$ and the Boussinesq approximation is used, there is a linear relation between the velocity and density fluctuations, corresponding to internal waves. These density perturbations are sourced by the entropy gradient present in the system. Zhuravleva et al. (2014a) showed that the linear relation may extend to the non-linear regime, such that $\left(\delta \rho_{k} / \rho\right)^{2} \approx \eta^{2}\left(v_{k} / c_{s}\right)^{2}$, where $\delta \rho_{k}$ and $v_{k}$ are some suitably defined fluctuation amplitudes at spatial scales $k^{-1}$ and $\eta \sim 1$ when the one dimensional velocity component is considered. Moreover, as the turbulent cascade proceeds to smaller scales, the role of buoyancy becomes negligible below the so-called Ozmidov scale. On these scales and under the above approximations, 
the entropy behaves as a passive scalar advected by the velocity field and, according to the Obukhov-Corrsin theory, the velocity and the scalar power spectra should have similar slopes, while the relative normalization is set by the coefficient $\eta$. Tests using relaxed clusters from cosmological simulations show $\eta \approx 1$, albeit with a $30 \%$ scatter.

Observationally, there are a few main hurdles associated with the X-ray fluctuation power spectral analysis. First, the choice of "unperturbed" model is not unique, and there is no perfect scale separation between the measured perturbations and the model. Thereby, the amplitude of density fluctuations measured on large scales may be affected by the choice of unperturbed model. Second, the final dynamic range of the observed power spectra is usually no larger than 10-30. Third, thermodynamic variables in full ICM hydrodynamics are not perfect scalars, thus at small scales (large $k$ modes) deviations from the approximate linear conversion are expected (Fig. 66). Keeping in mind such uncertainties and caveats, using the above relation, velocity power spectra have been retrieved from the observed density power spectra in the cool cores of several galaxy clusters.

Deep Chandra observations of Perseus and Virgo show that the hot gas in the core regions of these clusters is disturbed (Fig. 7) most likely by the activity of a powerful AGN (Forman et al. 2007, Fabian et al. 2011) residing in the center of both systems. This suggests that the gas may be turbulent. Zhuravleva et al. (2014b) used the power spectra of gas density fluctuations in these two galaxy cluster cores to report the first constraints on the corresponding velocity power spectra (for details of the analysis, uncertainties, and spectra in all regions in Perseus see Zhuravleva et al. 2015). Later work confirmed that about $80 \%(50 \%)$ of the total variance of perturbations in the Perseus (Virgo) core have isobaric nature, i.e. are consistent with subsonic gas motions in pressure equilibrium and/or gas cooling (Arévalo et al. 2016 Zhuravleva et al. 2016, Churazov et al. 2016). Walker et al. (2015) measured velocity power spectra also in the core of the Centaurus cluster. The velocities of gas motions inferred in this way vary between $\sim 80$ and $\sim 160 \mathrm{~km} / \mathrm{s}$ on spatial scales of $\sim 10-30 \mathrm{kpc}$ in the Perseus core, between $\sim 40$ and $\sim 80 \mathrm{~km} / \mathrm{s}$ on $\sim 2-10 \mathrm{kpc}$ scales in the Virgo core, and between $\sim 100$ and $\sim 150 \mathrm{~km} / \mathrm{s}$ on scales of $\sim 4-10 \mathrm{kpc}$ in Centaurus. Recently, velocity power spectra have been measured based on X-ray surface brightness fluctuations in several other nearby, cool core clusters that have deep Chandra observations (Zhuravleva et al. 2018). These constraints are shown in Figure 8. The typical velocities are consistent with those in Perseus, namely, between $\sim 100$ and $150 \mathrm{~km} / \mathrm{s}$ on scales $<50 \mathrm{kpc}$, and could be up to $\sim 300 \mathrm{~km} / \mathrm{s}$ on scales $\sim 100 \mathrm{kpc}$.

Hofmann et al. (2016) analyzed deep Chandra observations of 33 wellknown clusters, focusing instead on fluctuations of the projected thermodynamic quantities (the so-called pseudo density, temperature, pressure, entropy) within the core and outskirt $(>100 \mathrm{kpc})$ regions. They argue that the observed fluctuations correspond to a sample averaged 1D Mach number of $0.16 \pm 0.07$.

Khatri and Gaspari (2016) presented a study of thermal Sunyaev Zel'dovich (SZ) fluctuations in a hot (Coma) cluster with Planck, thus providing con- 


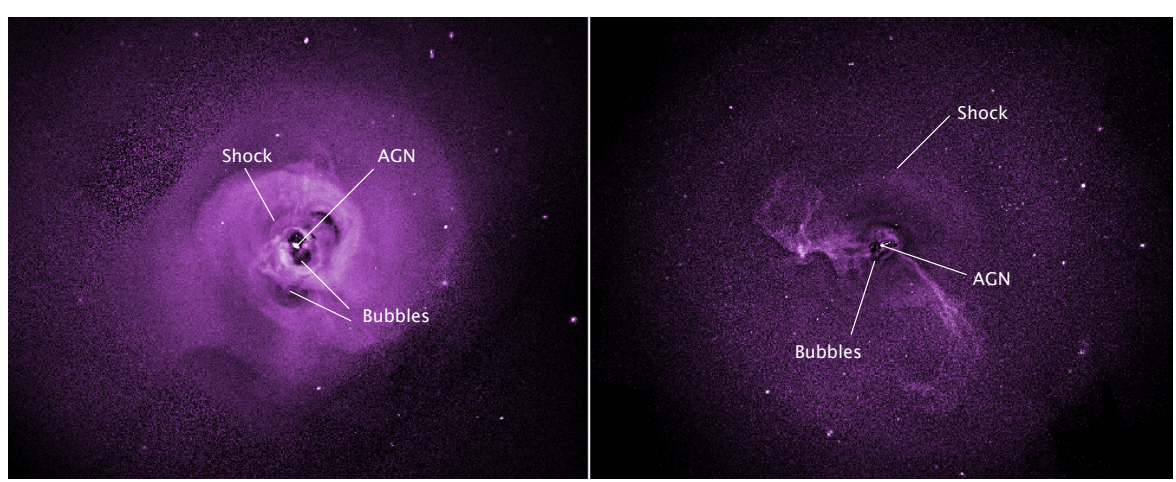

Fig. 7 Chandra residual images of the cool cores in the Perseus (left) and Virgo (right) galaxy clusters. Adapted from NASA/CXC/Stanford/Zhuravleva et al.
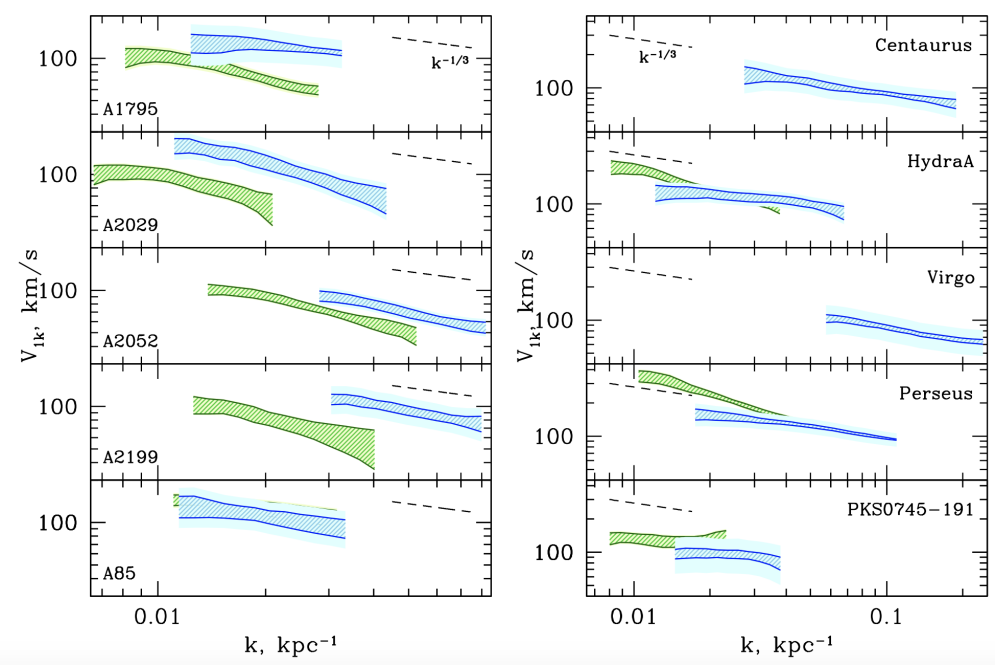

Fig. 8 Amplitude of the one-component velocity of gas motions versus wavenumber $k=1 / l$ determined from the analysis of X-ray surface brightness fluctuation power spectra for a sample of galaxy clusters (from Zhuravleva et al. 2018). Blue: velocities measured in the inner, half-cool-core regions. Green: same in the outer annulus of the cool core. Dashed lines show the slope of the amplitude for pure Kolmogorov turbulence (with arbitrary normalization). Hatched regions: velocities calculated using the mean sound speed in the gas. Solid regions: the spread of the velocity if maximal and minimal values of the sound speed are used.

straints on the turbulent power at the $500 \mathrm{kpc}$ injection scale (mainly associated with mergers) with retrieved 3D Mach number up to 0.8. The SZ power spectrum directly probes pressure fluctuations, which are thus complementary to the small-scale X-ray density fluctuations. Indeed, this study showed that the Coma X-ray power spectrum (with amplitude of $\sim 10 \%$; Churazov et al. 2012 Gaspari and Churazov 2013) can be extended and joined with the large-scale SZ power spectrum (with amplitude of $>30 \%$ ). Moreover, the 


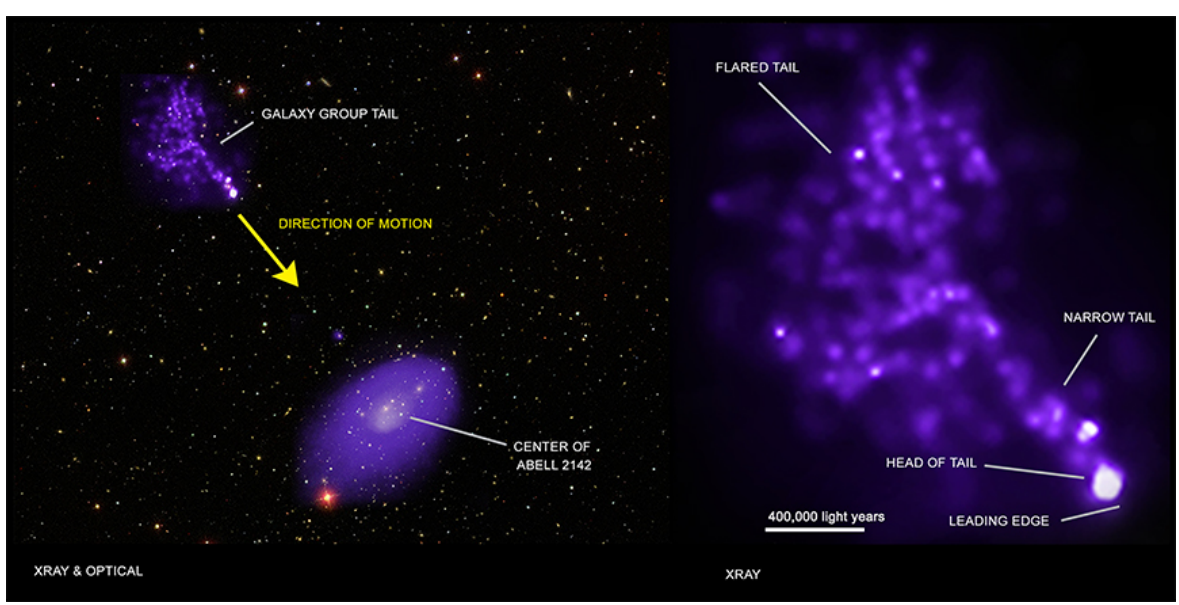

Fig. 9 The ram-pressure stripped tail of the A2142 infalling group, generating a cascade of turbulent motions with Mach numbers of 0.1-0.25 estimated from the surface brightness fluctuation analysis. Credit:X-ray: NASA/CXC/Univ. of Geneva, D. Eckert. Optical: SDSS provided by CDS through Aladin.

observed Coma (X-ray) power spectrum shows a slope consistent with the Kolmogorov value $\left(A_{\delta} \propto k^{-1 / 3}\right)$, indicating a highly suppressed thermal conductivity $\left(f \approx 10^{-3}\right)$. Other studies focused on measuring perturbations on large spatial scales via X-ray images have been carried out by Shibata et al. (2001); Finoguenov et al. (2005); Kawahara et al. (2008); Gu et al. (2009).

This method has also been used to investigate the connection between turbulence and particle re-acceleration in radio haloes. Eckert et al. (2017a) combined for the first time the large-scale power spectrum amplitude of X-ray fluctuations with the $1.4 \mathrm{GHz}$ radio power in over 50 clusters. Remarkably, those clusters hosting giant radio halos follow the relation $P_{\text {radio }} \propto \sigma_{v}^{3}$ defined by the turbulent energy flux rate. Bonafede et al. (2018) used the spectrum of density fluctuations from Chandra to suggest a different ratio of kinematic over thermal energy in the regions with and without extended radio halo in MACS J0717.5+3745, further strengthening the turbulent re-acceleration scenario. For a comprehensive review of the role of turbulence in magnetic field amplification and cosmic ray acceleration, we refer the reader to the chapters by Donnert et al. (2018) and van Weeren et al. (2019), included in this volume.

\subsubsection{Further considerations based on X-ray morphology}

In addition to analysing the power spectra of small-scale X-ray surface brightness fluctuations, the morphology and thermodynamical properties of specific substructures such as cold fronts or ram-pressure stripped tails seen on larger scales can provide additional information about the line-of-sight motions and the presence of turbulence or its suppression, or modification, by ICM viscosity, thermal conductivity, or magnetic fields. 
It was suggested early on that the ratio of thermal pressures at the stagnation point of a cold front and in the free stream region could be used to determine the velocity of the cold cloud with respect to the surrounding ICM (Vikhlinin et al. 2001a). This typically lead to near-sonic Mach numbers inferred from the observations (Vikhlinin et al. 2001a: Mazzotta et al. 2003 O'Hara et al. 2004, Machacek et al. 2005), although a few cases with small relative velocities (consistent with zero) were also identified (Markevitch et al. 2001: Tanaka et al.|2006). The challenge here is that the gas parameters at the stagnation point usually cannot be measured directly, because the stagnation region is physically small and its X-ray emission is strongly affected by projection. Away from the stagnation point, the strong tangential shear expected to be associated with cold fronts should promote the growth of hydrodynamic instabilities which can be a source of turbulence in the ICM. Initially, cold fronts appeared remarkably sharp, both in terms of the density and the temperature jumps, suggesting that the cold front could be stabilised by a layer of magnetic field parallel to the discontinuity (e.g. Vikhlinin et al. 2001b), or viscosity (e.g. Roediger et al.|2013). Recent, deeper observations have revealed evidence for the onset of Kelvin-Helmholz (KH) instabilities in a number of systems (Roediger et al. 2012b; Roediger et al. 2012a; Werner et al. 2016, Walker et al. 2017). Notably, in the cases of the cold fronts in A3667 and the Perseus Cluster, Ichinohe et al. (2017) and Ichinohe et al. (2019) calculated the thermal pressure deficit associated with candidate $\mathrm{KH}$ rolls compared to the ambient ICM; assuming this deficit is solely due to turbulent pressure support, they report $v_{\text {turb }} \sim 300-400 \mathrm{~km} / \mathrm{s}$ in both cases. Although these estimates are currently uncertain to within a factor of a few, this illustrates the power of combining high spatial resolution cold front morphology with direct measurements of line broadening from future high spectral resolution X-ray telescopes to constrain the microphysics of the ICM. Based on the onset of $\mathrm{KH}$ instabilities in A3667, Ichinohe et al. (2017) put an upper limit on the effective ICM viscosity at $5 \%$ of the isotropic Spitzer value.

Comparing tailored simulations and deep observations of the stripped galaxy M89 in Virgo, Roediger et al. (2015b) and Kraft et al. (2017) do not find indications of viscous or other suppression or mixing of cold stripped gas with the hotter ICM in the wake of this galaxy. Similarly, the wake of NGC 1404 seems to be well mixed too, indicating that the effective viscosity is at most $5 \%$ of the Spitzer value (Su et al. 2017b). However, to apply results from generic, idealised simulations to specific objects, the dynamical conditions of the stripped subcluster in question (e.g., the initial gas contents or the merger stage) need to be taken into account. Eckert et al. (2017b) used the X-ray surface brightness fluctuation analysis discussed in the previous subsection to study the kinematics within the tail and wake of the ram-pressure stripped group observed falling into Abell 2142 (Fig. 9). The retrieved turbulent Mach numbers generated by this infall are in the range $\mathrm{Mach}_{3 \mathrm{D}} \sim 0.1-0.25$, which coincidentally seems to be similar to the level of subsonic turbulence driven in the ICM cores by the AGN feedback cycle. The shallow slope of the fluctu- 
ation power spectrum in Abell 2142 indicates that transport properties (such as thermal conduction) are far below the Spitzer value.

\subsubsection{The kinematic Sunyaev-Zel'dovich effect}

In addition to the widely used thermal Sunyaev-Zel'dovich (SZ) effect, wherein cosmic microwave background (CMB) photons passing through the ICM are inverse Compton scattered from the low-frequency region of the blackbody spectrum to substantially higher energies, gas bulk motions in the ICM can lead to an additional distortion of the CMB spectrum (Sunyaev and Zeldovich 1980), expressed as

$$
\frac{\Delta T}{T}=-\sigma_{\mathrm{T}} \int n_{e} \boldsymbol{\beta} d \ell .
$$

Here, $\beta \equiv v \mathbf{z} / c$ is the unitless (bulk) velocity, and $\sigma_{\mathrm{T}}$ is the Thompson crosssection. This kinematic SZ (kSZ) signal is subdominant to the thermal SZ, unless the gas velocity reaches a few tenths of a percent of the speed of light $\left(1000 \mathrm{~km} \mathrm{~s}^{-1}\right.$, see e.g. Birkinshaw 1999). In addition, since this effect manifests itself only as an overall shift in the CMB temperature, it is indistinguishable in principle from temperature variations corresponding to primary CMB fluctuations, making its direct detection rather challenging. However, on small angular scales where the contribution from primary CMB anisotropies becomes small, the kinematic SZ (kSZ) effect can allow measurements of the gas bulk velocities and especially velocity gradients in the ICM.

The first spatially resolved map of the $\mathrm{kSZ}$ signal in a galaxy cluster was presented recently by Adam et al. (2017), using data obtained with the NIKA camera at the IRAM $30 \mathrm{~m}$ telescope. They observed the merging cluster MACS J0717.5+3745 and detected a dipolar structure in the kSZ signal, with peaks that correspond to two subclusters moving away and toward us at velocities of several thousand $\mathrm{km} / \mathrm{s}$. This opens the way for future high-resolution SZ observations promising to provide much more sensitive constraints on gas motions in the ICM via the $\mathrm{kSZ}$ effect.

\subsubsection{Optical/sub-mm line-emission}

Central cluster galaxies in cool core clusters are frequently observed to host optical line-emitting filaments (see e.g. Crawford et al. 1999, and citations thereof). More recently, these structures have been found to be multi-phase (e.g. Edge 2001; Salomé et al. 2006; Sparks et al. 2009; Salomé et al. 2011; Mittal et al. |2011, 2012 |Werner et al. 2014; Tremblay et al. 2015; Anderson and Sunyaev 2018), consisting of gas from $<100 \mathrm{~K} 10^{4} \mathrm{~K}$ as detected through molecular $\mathrm{CO}$, ionised $\mathrm{C}$ and $\mathrm{O}$, and $\mathrm{Ly}-\alpha$ emission lines, much of which has been observed to be extended and filamentary. In particular, the high spatial and spectral resolution of ALMA has been instrumental in linking the warm ionized and cold molecular gas phases and uncovering the total mass (most of which is in the cold phase) within the filamentary systems (e.g. David 

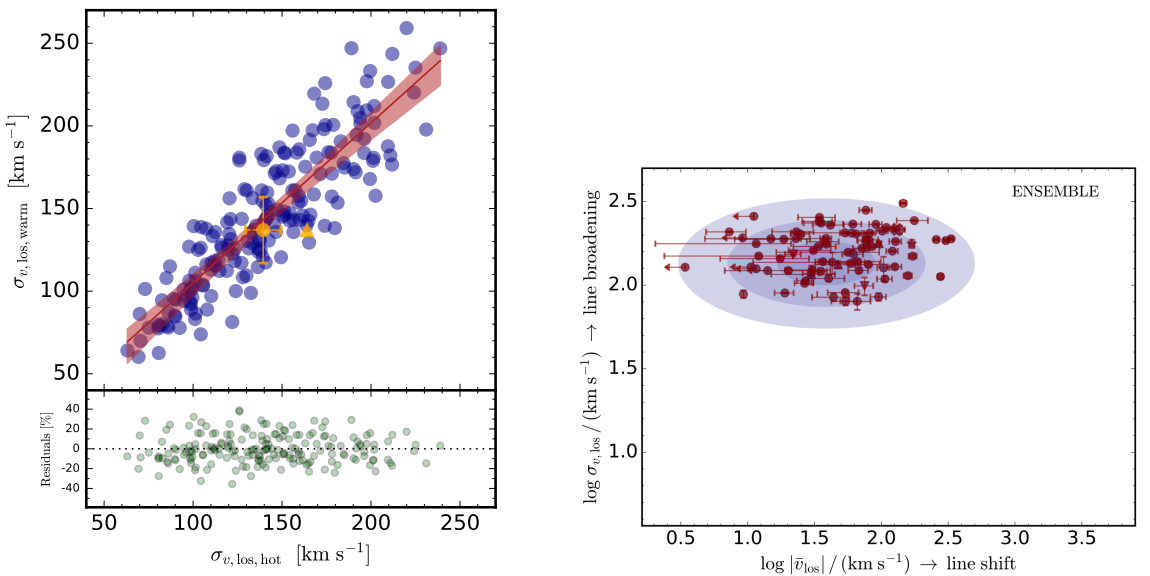

Fig. 10 Left: Correlation between the luminosity-weighted velocity dispersion of warm $\left(\sim 10^{4} \mathrm{~K}\right)$ gas and the hot ICM $(0.3-8 \mathrm{keV})$ in the cooling core $(\lesssim 50 \mathrm{kpc})$. The red line and bands show the best-fit linear regression and the associated $99 \%$ confidence interval. The yellow point shows the observational constraints from the Perseus cluster combining the SITELLE $\mathrm{H} \alpha+[\mathrm{NII}]$ data with the Hitomi measurements (Section 3.2. Right: velocity dispersion versus the magnitude of the LOS velocity for the warm and cold gas phases, comparing the observational data (red points) with the predictions from numerical simulations (1-3 $\sigma$ contours) for the ensemble detections. Adapted from Gaspari et al. (2018).

et al. 2014, McNamara et al. 2014, Vantyghem et al. 2016, Russell et al. 2016, 2017a|b; Temi et al. 2018; Pulido et al. 2018; Simionescu et al. 2018; Tremblay et al. 2018). The excitation mechanisms of this multi-phase gas have been well studied, but are beyond the scope of this review.

The frequency of ionized filaments in the central galaxies of cool core clusters compared with those of non-cool core clusters imply they are connected with the hot cluster atmosphere either by cooling directly into filaments, being uplifted into the hot atmosphere from the central regions of the galaxy by the action of AGN feedback, or a combination of the two (see Section 2.4). The morphologies and kinematics of these filaments can provide insight into the ICM velocity structure and physical properties (Hamer et al. 2016: McDonald et al. 2012, Russell et al. 2016, 2017a b, Tremblay et al.|2018). It was argued early on that the thin and long shape of ionised and molecular gas filaments implied a quasi-laminar or viscous flow with little small scale turbulence (e.g. Fabian et al. 2003b, Hatch et al. 2005). However, initial debates regarding the origin of these structures made it unclear whether and to what extent their dynamics faithfully traced the motions of the ICM, or if these filaments were rather immune to their ambient medium like "tree logs in the wind". Recent ALMA observations have shown that lifting the observed amount of molecular gas out of the cluster cores requires an infeasibly efficient coupling between the molecular gas and the radio bubbles (McNamara et al. 2014: Russell et al. 2016, 2017b); this, together with the fact that the velocities of the molecular gas clouds are well below their free-fall speeds, favour a scenario in which 
the observed filaments are the result of cooling instabilities in the X-ray gas triggered in situ by disturbances associated with the AGN feedback process (Gaspari et al. 2013a; Voit et al. 2015. McNamara et al. 2016). If the lineemitting filamentary nebulae indeed originate from the cooling of the ICM, it is naturally expected that their velocity structure should trace that of the ambient X-ray plasma.

By using high-resolution hydrodynamical simulations including self-regulated AGN jet feedback, Gaspari et al. (2018) showed that all gas phases in cooling cores are tightly linked in terms of the ensemble LOS velocity dispersion (see Fig. 10), indicating that the spectroscopic measurements of the velocity dispersion in the warm/cold gas measured in wide (arcmin) apertures are reliable tracers of the velocity dispersion of the hot X-ray emitting gas. The retrieved best-fit correlation of the ensemble warm-gas velocity dispersion is $\sigma_{v \text {, warm }}=0.97_{-0.02}^{+0.01} \sigma_{v, \text { hot }}+8.3_{-5.1}^{+3.5}\left(\mathrm{~km} \mathrm{~s}^{-1}\right)$. The authors show that observations of massive galaxies and central group and cluster galaxies have little scatter in their ensemble line widths suggesting a common level of turbulence in the hot gas of $100-250 \mathrm{~km} / \mathrm{s}$, consistent with the direct line width measurement from hot gas in the Perseus cluster (Hitomi Collaboration 2016) and those of density fluctuations measured from X-ray images (see Section 3.1.4). This study also showed that the opposite, pencil-beam approach is key to constrain the small-scale turbulence and bulk motions of single elements: the smaller the beam, the better we can probe the single cold/warm clouds raining via $\mathrm{CCA}$ toward the $\mathrm{SMBH}$, which will trigger the subsequent feedback event. Most of the detected clouds and filaments have virial parameter $\gg 1$, i.e., they are highly dynamically supported as they inherit the velocity dispersion during the turbulence cascade from the parent hot halo (Gaspari et al. 2017).

Another, potentially exciting, avenue to constrain gas motions of the ICM arises from heavy element hyperfine structure transitions in the radio probing $10^{58} \mathrm{~K}$ (Sunyaev and Churazov 1984) and coronal emission lines (Anderson and Sunyaev 2016). So called, due to their abundance in the optical spectra of the Sun, coronal lines originate from gas at $\sim 10^{56} \mathrm{~K}$. This phase of gas provides the missing link between the $\lesssim 10^{4} \mathrm{~K}$ filaments and the hot ICM, offering a direct means of measuring the rate at which the ICM is condensing (Cowie 1981). Moreover, the comparatively high spatial resolution of optical and radio measurements compared with the X-ray data would enable us to trace the hot gas velocities at sub-arcsecond scales.

Graney and Sarazin (1990), Sarazin and Graney (1991), Voit et al. (1994) and most recently, using non-equilibrium cooling, Chatzikos et al. (2015) have modelled coronal-line emission in conditions appropriate to those of the cores of galaxy clusters. Despite evidence for gas at these temperatures from X-ray and UV lines (e.g. Sanders et al. 2010b; Pinto et al. 2014; Bregman et al. 2006; Anderson and Sunyaev 2018), hyperfine structure transitions of heavy elements have never been observed from cooling gas in galaxy clusters and optical coronal lines remain elusive with mostly upper limits reported (e.g. $\mathrm{Hu}$ et al. 1985; Heckman et al. 1989, Anton et al. 1991, Donahue and Stocke 1994; Yan and Cohen 1995). Canning et al. (2011) report a 6.3 sigma detection of 
Fe $\mathrm{X}$ emission, a tracer of $10^{5} \mathrm{~K}$ gas in the central galaxy of the Centaurus cluster with a velocity width of $300-650 \mathrm{~km} / \mathrm{s}$, though they conclude this is likely the result of gas heated by interaction with the radio lobes of the AGN rather than cooled from the hot ICM.

\subsection{Recent progress using micro-calorimeter X-ray spectroscopy}

The Hitomi Soft X-ray Spectrometer (SXS) observations of the Perseus cluster allowed us to perform the first precise, direct measurements of velocity dispersions and bulk motions in the ICM. The exquisite spectral resolution of this detector (a factor of more than 20 better than conventional CCDs), combined with its non-dispersive nature that circumvents the line broadening for extended sources affecting the XMM-Newton RGS, provided a break-through in our understanding of the dynamics of the hot, diffuse ICM. The line broadening due to turbulent velocities is clearly resolved, above the instrumental and thermal broadening (Fig 11).

The initial Hitomi results yielded a velocity dispersion of $164 \pm 10 \mathrm{~km} / \mathrm{s}$ (at $90 \%$ confidence) in a region located 30-60 kiloparsecs from the central nucleus, and a hint of a slightly higher turbulent velocity in the immediate vicinity of the AGN (Hitomi Collaboration 2016). A subsequent, more in depth analysis presented in Hitomi Collaboration (2018a) accounted for the smearing effects of the Hitomi PSF and revealed that the velocity dispersion reaches maxima of approximately $200 \mathrm{~km} \mathrm{~s}^{-1}$ toward the central AGN and toward the AGN inflated north-western 'ghost bubble (left panel of Fig. 12). Elsewhere within the observed region, the velocity dispersion appears constant around $100 \mathrm{~km}$ $\mathrm{s}^{-1}$. There is a line-of-sight velocity gradient with a $100 \mathrm{~km} \mathrm{~s}^{-1}$ amplitude across the cluster core, consistent with large-scale sloshing of the core gas (right panel of Fig. 12).

In principle, the observed line widths quoted above can be due both to small-scale near-isotropic turbulence and to a superposition of laminar motions along the line of sight (see discussion in Section 1.2). It is important to note therefore that, in addition to the measurements of line widths and shifts quoted above, the Hitomi data allows for several other tests that can reveal the nature and driving scale of gas motions in the Perseus cluster core.

The first of these tests relies on measurements of the shapes of strong spectral lines (as suggested by Zhuravleva et al. 2011, Shang and Oh 2012, 2013). While turbulence on small scales will increase the observed line widths of well-resolved optically thin emission lines maintaining a Gaussian shape, gas motions on large scales will shift the line centroids. The superposition of large scale motions within the spectral extraction area should therefore lead to non-Gaussian features in the observed line profiles (e.g. Inogamov and Sunyaev 2003). Hitomi Collaboration (2018a) report a lack of evidence for non-Gaussian line shapes, indicating that the observed velocity dispersion is dominated by small scale motions with a turbulent driving scale likely below $100 \mathrm{kpc}$, which is consistent with the size of the AGN jet inflated bubbles. 


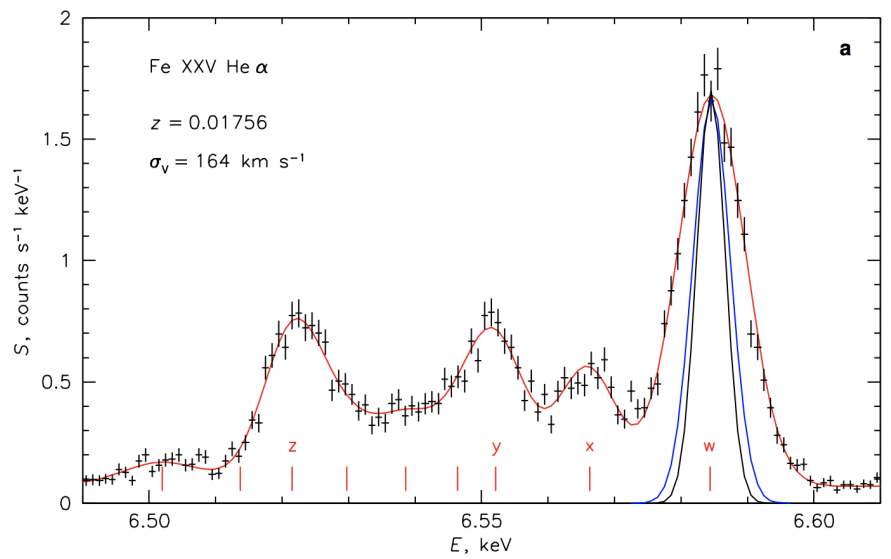

Fig. 11 Spectrum of the core of the Perseus Cluster obtained by the Hitomi SXS, focusing on the Fe XXV He- $\alpha$ line complex. Instrumental broadening with (blue line) and without (black line) thermal broadening are indicated, demonstrating that the line width due to turbulent velocities is clearly resolved for the first time. From Hitomi Collaboration (2016).
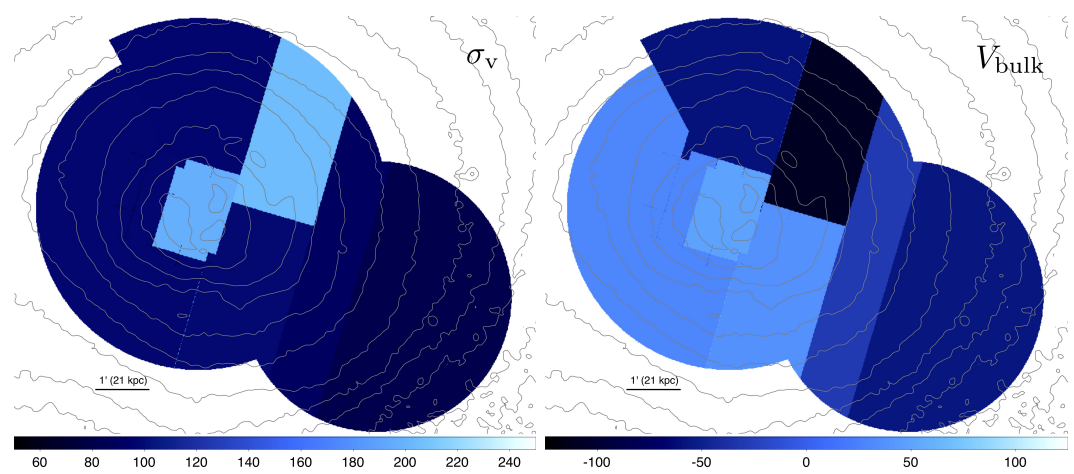

Fig. 12 PSF corrected maps of the velocity dispersion $\left(\sigma_{\mathrm{v}}\right.$, left $)$ and bulk velocity $\left(v_{\mathrm{bulk}}\right.$, right) in the core of the Perseus Cluster obtained from Hitomi. The bulk velocity is expressed relative to $z=0.017284$ (heliocentric correction applied). The unit of the values is $\mathrm{km} \mathrm{s}^{-1}$. The Chandra X-ray contours are overlaid. Adapted from Hitomi Collaboration (2018a).

The second test relies on the suppression of lines with high oscillator strengths by resonance scattering (Gilfanov et al. 1987, Churazov et al. 2010. and Section 3.1.3). Line broadening and resonance scattering as a function of radius have a different dependence on the directionality of gas motions and therefore, in principle, they allow us to reveal and place constraints on the anisotropy of gas motions in bright cluster cores (Rebusco et al. 2008, Zhuravleva et al. 2011).

The Hitomi SXS observation of the Perseus cluster allowed the robust measurement of resonant scattering in the strongest X-ray line of the spectrum, the Fe XXV He $\alpha$ line (w). The measured flux suppression in this line is a 

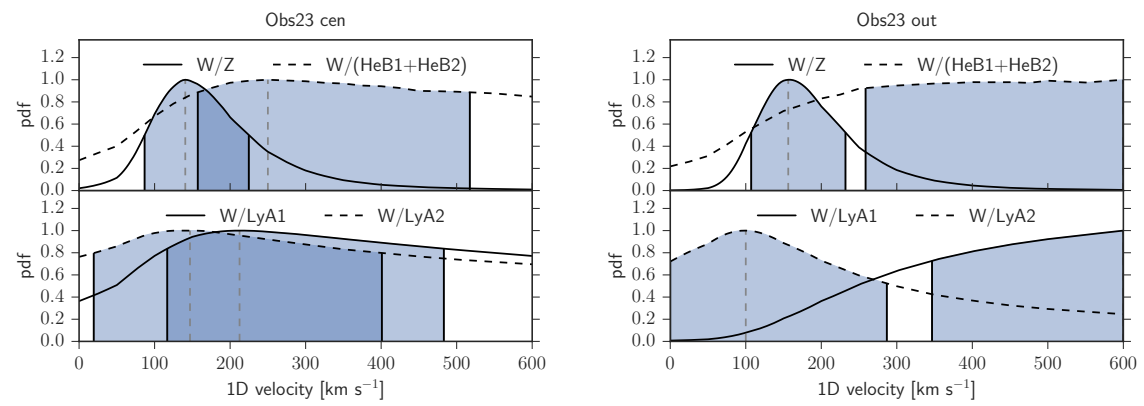

Fig. 13 Probability distributions for the turbulent velocities in the core of the Perseus Cluster inferred from measurements of the resonance scattering effect obtained by Hitomi. The flux ratios of the resonant Fe XXV He $\alpha$ line ('W') with respect to various emission lines that are approximately optically thin (Fe XXV He $\alpha$ forbidden line 'Z', Fe XXV He $\beta$, and two Fe XXVI Ly $\alpha$ lines) are compared to predictions from radiative transfer simulations to obtain these constraints. The left panel shows the inner $\sim 30 \mathrm{kpc}$ region, while the right panel shows a region $\sim 30-100 \mathrm{kpc}$ away from the cluster center. Especially in the outer region, uncertainties in the predictions of line emissivities in optically thin plasma models have a significant effect on the inferred turbulent velocities. Adapted from Hitomi Collaboration (2018b).

factor of $\sim 1.3$ in the inner $\sim 40 \mathrm{kpc}$, and decreases to $\sim 1.15$ in the region $\sim 30-100 \mathrm{kpc}$ away from the cluster center (Hitomi Collaboration 2018b). Comparing these suppressions with the results of radiative transfer simulations in the Perseus cluster, turbulent velocities of $150_{-56}^{+80} \mathrm{~km} / \mathrm{s}$ and $162_{-50}^{+78} \mathrm{~km} / \mathrm{s}$ have been measured in both regions (Fig. 13). The results are consistent with the direct measurements from line broadening (Hitomi Collaboration 2018a), suggesting a lack of anisotropy in the gas motions (in agreement with the Gaussian shape of strong emission lines). However, both the systematic and the statistical uncertainties remain large. For instance, different choices of reference optically thin lines with respect to which the suppression of the Fe XXV He $\alpha$-w is calculated can affect the inferred turbulent velocities (Fig. 13). In order to provide meaningful constraints on the anisotropy of gas motions, both further improvements in the predictions of line emissivities in plasma models and deeper observations will be necessary.

It is moreover interesting to compare the velocities obtained from the surface brightness fluctuation analysis and high-resolution Hitomi spectra in Perseus, as a gauge for the validity of the method discussed in Section 3.1.4. The total line-of-sight velocity dispersion observed by Hitomi from the region $r \lesssim 100 \mathrm{kpc}$, can be estimated as $V_{1 \mathrm{D}}=\sqrt{\sigma^{2}+\Delta V^{2}}$. Here $\sigma$ and $\Delta V$ are, respectively, the line broadening measured in individual pixels and the centroid shift across the region (see ZuHone et al. 2018, for detailed discussion). Taking into account the radial distribution of the gas contributing to the line flux, one can interpret the Hitomi value as an estimate of the velocity amplitude on scales of $\sim 60 \mathrm{kpc}$. At this scale, the velocity dispersion measured by Hitomi is in agreement with the fluctuations analysis amplitude (Zhuravleva et al. 2014b, 2018; Hitomi Collaboration 2018a). Moreover, Figure 10 reveals 
an excellent agreement between the Hitomi velocity dispersion for the Perseus Cluster and the line width of the ensemble warm $\mathrm{H} \alpha+[\mathrm{NII}]$ phase obtained from SITELLE data (Gaspari et al. 2018). In a subsequent work, GendronMarsolais et al. (2018) studied several smaller-scale patches of the NGC 1275 nebula detected with SITELLE, finding that, while the velocity dispersions of the filamentary nebula surrounding NGC 1275 are indeed as low and uniform as the Hitomi measurements of the ICM, there is no correlation between the bulk velocities of the different phases.

\section{Implications for distinguishing the source of turbulent motions and constraining the ICM viscosity}

The publication of the precise measurements of the ICM dynamics in the core of the Perseus Cluster obtained by Hitomi has sparked a lively debate in the community, with questions revolving around

1. what is the main physical mechanism that is driving the gas motions?

2. is the observed level of turbulence sufficient to balance radiative cooling?

3. what, if any, are the physical implications for constraining the effective viscosity of the ICM?

In this section, we summarise and discuss the various proposed interpretations.

In terms of the source of gas motions, it is clear from Section 2 that we are looking at a superposition of various physical processes which all predict roughly similar turbulent velocities within the central region of clusters (at the level of a few hundred $\mathrm{km} / \mathrm{s}$ ). Based on the average level of turbulent motions alone, it is then difficult, if not impossible, to distinguish which of these different processes is the dominant one. This question can be addressed through a spatially-resolved comparison of the line shifts and widths as a function of radius or in regions of various X-ray morphology (i.e. associated with cavities or aligned with existing cold fronts).

The peaks in $\sigma_{v}$ seen in the Hitomi observations of the Perseus Cluster appear to indicate that gas motions are driven both at the cluster center by the current AGN inflated bubbles and by the buoyantly rising ghost bubble with a diameter of $\sim 25 \mathrm{kpc}$ (Hitomi Collaboration 2018a). This appears to contradict models in which gas motions are sourced only at the center (during the initial stages of bubble inflation) or only by structure formation.

At the same time, surface brightness features in the Perseus cluster indicate the presence of gas sloshing (Churazov et al. 2003, Simionescu et al. 2012, Walker et al. 2017) which will also drive a part of the observed motions (see Section 2.2). The relative uniformity of the velocity dispersion, apart from the regions associated with AGN bubbles, may be consistent with sloshinginduced turbulence (ZuHone et al. 2013). In this case, the shearing motions associated with gas sloshing are also expected to contribute to the velocity dispersion observed throughout the investigated area and thus the true turbulence might be even weaker. The spatial coverage of the Hitomi pointings, 
as well as the spatial resolution, limits our ability to fully disentangle the impact of the AGN and ongoing gas sloshing on the velocity structure of the ICM. Using the density fluctuations in deep Chandra observations to estimate the turbulent velocities instead, Walker et al. (2018b) suggest that these are broadly consistent with being produced by sloshing alone outside the central $60 \mathrm{kpc}$.

The problem becomes even more complex when the expected velocity field from cosmological large-scale structure formation is taken into account, in addition to AGN feedback and idealised gas sloshing. Lau et al. (2017) performed an analysis of mock Hitomi observations of clusters from cosmological simulations and isolated clusters with AGN feedback (from Gaspari et al. 2012a). They concluded that cosmic accretion and mergers could produce line-of-sight velocity dispersions and line shifts compatible with the Hitomi observations of Perseus, while AGN feedback is able to produce velocity dispersion measurements which are compatible with the Hitomi observations, but not the core-scale velocity gradient, since the turbulence driven by AGN feedback is too stochastic. Bourne and Sijacki (2017) reached similar conclusions using mock Hitomi observations of simulated isolated clusters with AGN-driven jets and models which included realistic cosmic substructure: AGN feedback can produce the observed line widths, but producing the observed line shifts requires gas motions driven by mergers.

Spatially-resolved high-resolution spectroscopy covering a larger area of the Perseus Cluster core, as well as a number of other systems, will be crucial to reach an agreement on which physical process is the main source of gas motions, and how this changes as a function of radius.

In terms of the feasibility of balancing radiative cooling through turbulent dissipation, Zhuravleva et al. (2014b) first showed, using the surface brightness fluctuation analysis, that turbulent heating is not only sufficient to offset radiative cooling but indeed appears to match it locally at each radius, for 7 different annuli in Perseus and 4 in M87. The fact that the turbulent velocities inferred from this surface brightness fluctuation analysis were found to be in very good agreement with the direct constraints from the Hitomi SXS (Section 3.2 lends further support to this interpretation. On the other hand, Fabian et al. (2017) and Bambic et al. (2018) argue that this low-velocity turbulence cannot spread far across the cooling core during the fraction of the cooling time in which it must be replenished, and hence conclude that another heating mechanism such as sound waves must dominate. However, this argument can be circumvented if the turbulence is driven in situ by the rising bubbles, as suggested by the higher velocity dispersion associated with the ghost cavity in the Perseus Cluster (Hitomi Collaboration 2018a). Ultimately, tests based on the radial dependence of the line widths and line shapes discussed in Section 2.4 are expected to shed more light on this issue in the future.

In terms of the microphysical properties of the ICM, it is at first sight tempting to interpret the relatively small gas velocities measured by Hitomi in a cool core cluster hosting strong signatures of sloshing activity as evidence for a high effective viscosity of the X-ray plasma. Indirect probes of the gas 
velocities in other cool core clusters, such as surface brightness fluctuations or the width of spectral lines from the warm (rather than hot) phase, similarly support relatively low turbulent velocities, as discussed above. To investigate this, ZuHone et al. (2018) performed two simulations of gas sloshing in a galaxy cluster core similar to the Perseus cluster, in the absence of AGN feedback, to study the effects of sloshing in isolation on the line-of-sight measurements of the velocity field; the simulations differed only in that the first was inviscid and the second was highly viscous with the isotropic Spitzer viscosity. ZuHone et al. (2018) found an orientation of the line of sight angle which reproduced both the spiral shape of the cold fronts and the velocity shear observed by Hitomi across the core region, and performed mock Hitomi observations. These observations were used to produce measurements of the line shift and width similar to those obtained for Perseus. Their results showed that at the $\sim 1$ ' spatial resolution afforded by Hitomi, it is very difficult to distinguish the velocity field of a highly viscous ICM from an inviscid one (see Figure 14). This is because the broadening of spectral lines is produced mainly by nearly core-size eddies which are only marginally affected by even the high level of viscosity employed in the simulation (ZuHone et al. 2016).

In addition, as mentioned above, numerical models of cosmic accretion and AGN feedback presented by Lau et al. (2017) and Bourne and Sijacki (2017) are able to roughly reproduce the level of turbulent motions observed by Hitomi without including viscosity explicitly in the calculation. Together with the ZuHone et al. (2018) results, this not only implies that gas motions from cosmic accretion/mergers/sloshing on the one hand and AGN feedback on the other are complementary drivers of the velocity field in cluster cores, but viscosity is not required to explain the level of gas motions seen in Perseus, provided that the system has not experienced a major merger recently. This is in line with the results discussed in Section 3.1.5. where the morphology of cold fronts and ram-pressure stripped tails suggest a low viscosity of the ICM for the cases of M89 (Roediger et al. 2015b; Kraft et al. 2017), NGC1404 (Su et al. 2017b), A2142 (Eckert et al.|2017b), and A3667 (Ichinohe et al. 2017).

Constraints on the microphysics of the ICM will require both a larger sample of clusters for which precise velocity measurements are available, but also an ensemble of observational strategies; while X-ray line broadening alone may be insufficient to obtain an estimate of the plasma viscosity, combining this information with measurements of resonance scattering and high-spatial resolution analysis of the X-ray morphology will provide a very powerful technique accessible to future missions, as discussed below.

\section{Future prospects}

Future X-ray missions, like XRISM, Athena and Lynx will measure radial profiles of the gas velocity dispersion and bulk velocities in many objects, allowing us to probe both the velocity amplitudes and scales (Zhuravleva et al. 2012, ZuHone et al. 2016, Roncarelli et al.|2018), and, therefore, systematically com- 


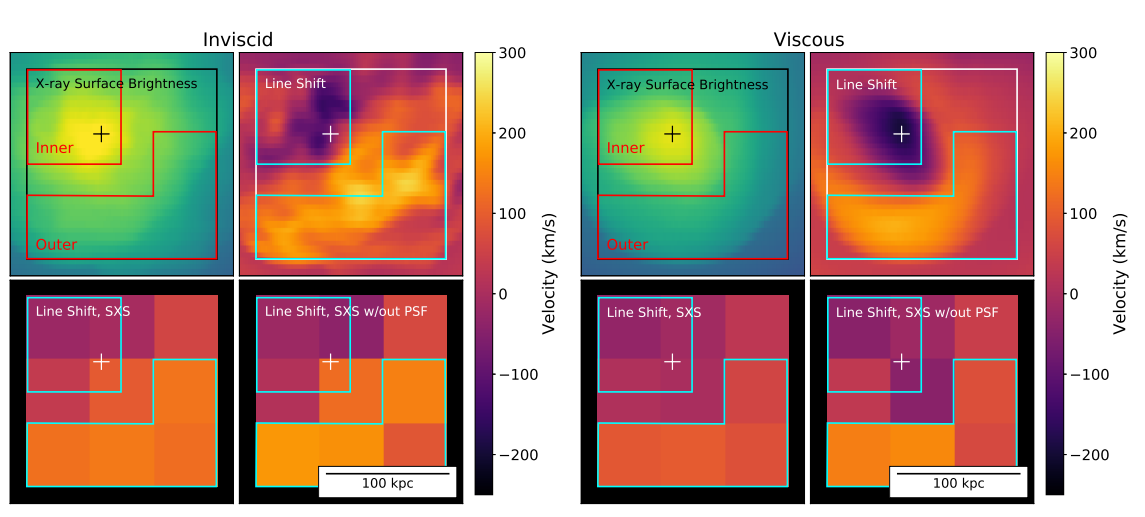

Fig. 14 Mock line shift maps for a Perseus-like cluster from ZuHone et al. (2018) from two hydrodynamic simulations, one inviscid and the other highly viscous. The top panels show the full simulation spatial resolution while the bottom show the Hitomi angular resolution. It is not possible to discern the difference in the velocity fields due to viscosity in the mock Hitomi observations.

pare the imaging and spectral techniques. We summarise below the progress expected from these future missions, together with the improvements expected for experiments in other wavebands.

\subsection{Measuring gas motions with XRISM}

The X-Ray Imaging and Spectroscopy Mission (XRISM), formerly XARM (the X-ray Astronomy Recovery Mission, Tashiro et al. 2018) is planned as a successor of the Hitomi satellite, and will carry a high spectral resolution X-ray microcalorimeter (Resolve), which is identical to the SXS. This will allow to extend detailed studies like that performed by Hitomi for the Perseus Cluster to many other systems, and to softer X-rays (the SXS was planned to cover energies down to $0.3 \mathrm{keV}$, but was limited to $>2 \mathrm{keV}$ because of the transmission of the Beryllium filter that was in place during the early phase of the operation). The progress expected for galaxy cluster observations, including the measurement of gas motions in the ICM, using Resolve is very similar to the science case for the SXS, summarised in Kitayama et al. (2014).

In terms of constraining the nature and origin of gas motions, as well as the microphysical properties of the ICM, XRISM is relevant for a number of complementary aspects. Firstly, Resolve will allow us to study the gas velocities in a larger sample of relaxed, cool-core clusters, and evaluate the impact of the central SMBH on the ICM dynamics. In a few cases (most notably, M87), the X-ray surface brightness features associated with the ongoing AGN feedback can be spatially resolved, and differential tests of the nature of gas motions corresponding to and offset from these AGN-related substructures can inform us about the mechanisms through which supermassive black holes drive turbulence in the ICM. 
Secondly, Resolve will provide further insights into the impact of minor and major mergers in determining the kinematics of the ICM. Differential tests of the velocity structure performed for regions located in- and outside cold fronts, or on/offset from bright ram-pressure stripped tails of infalling subhaloes will probe the importance of minor mergers. Major mergers of galaxy clusters will also be a promising target, due to the fact that they produce bulk motions up to several $1000 \mathrm{~km} \mathrm{~s}^{-1}$ and turbulent motions of $\sim 1000 \mathrm{~km} \mathrm{~s}^{-1}$. The strongest and the most interesting signals will be observed for mergers occurring predominantly along the line of sight. Due to the complex state of gas motions in mergers and projection effects (see Section 2.2), all these observations will have to be interpreted very carefully in combination with simulations.

$X R I S M$ measurements will likely focus on the inner regions of nearby massive galaxy clusters. A detailed characterisation of the gas velocity profile out to beyond $r \approx R_{2500}$ will require a significant investment of observing time, and can only be performed for a very limited sample of objects. However, if this time investment is made, Nagai et al. (2013) show that the analysis of mock SXS (analogous to Resolve) spectra of a relaxed galaxy cluster extracted from cosmological numerical simulations recovers the 3D (deprojected) massweighted velocity dispersion profile up to $r \approx R_{500}$ accurately, while slightly $\left(\sim 30-50 \mathrm{~km} \mathrm{~s}^{-1}\right)$ underestimating the projected mass-weighted gas velocity dispersion in a given radial bin. This difference occurs because the measured velocity is spectral-weighted, and hence the inner regions where the gas density is higher but the gas velocity is smaller carry a higher weight. Going forward, this synergy between numerical simulations, mock observations, and real data will need to be employed frequently in order to correctly interpret the measurements and uncover any potential biases or significant projection effects (see also Ota et al. 2018).

To effectively expand our knowledge of the ICM velocity structure towards the cluster outskirts, future missions beyond XRISM will be necessary. In addition to the significant progress expected to be obtained with Athena (see the next subsection), the Chinese-led Hot Universe Baryon Surveyor (HUBS 4 is another promising mission concept that is currently under consideration. With a large effective area $\left(\sim 1000 \mathrm{~cm}^{2}\right)$ and large field of view $(\sim 1$ square degree), moderate angular resolution of 1 arcmin, and high spectral resolution $(2 \mathrm{eV}$ at $0.6 \mathrm{keV}$ ) in the soft X-ray band (0.1 to $2 \mathrm{keV})$, HUBS is optimised to look for the missing baryons in intergalactic and circumgalactic space. However, its capabilities are also very well suited to provide robust measurements the widths and shifts of X-ray lines in the faint outskirts of nearby clusters of galaxies (see also the related chapter on "The physics of galaxy cluster outskirts", Walker et al. 2019, this volume).

\footnotetext{
4 http://heat.tsinghua.edu.cn/ ${ }^{\sim}$ hubs/en/index.html
} 


\subsection{Measuring gas motions with Athena/X-IFU}

Athena (Nandra et al. 2013) is the second large mission selected in the framework of the European Space Agency (ESA) Cosmic Vision program and it is scheduled for launch in the early 2030s. The mission will carry a highthroughput X-ray telescope with an unprecedented effective area of $1.4 \mathrm{~m}^{2}$ at $1 \mathrm{keV}$ and an angular resolution of 5 arcsec. The telescope will feed two instruments in the focal plane, the Wide-Field Imager (WFI) and the X-ray Integral Field Unit (X-IFU). The X-IFU (Barret et al. 2018) is an array of $\sim 4,000$ transition-edge sensors (TES) with a spectral resolution of $2.5 \mathrm{eV}$ at $7 \mathrm{keV}$ and covering a field of view of 5 arcmin diameter. The capabilities of $\mathrm{X}$-IFU will allow us to detect line shifts and broadening down to $\sim 20 \mathrm{~km} / \mathrm{s}$ and map them at a resolution of $5 \operatorname{arcsec}$ (see also Ettori et al. 2013).
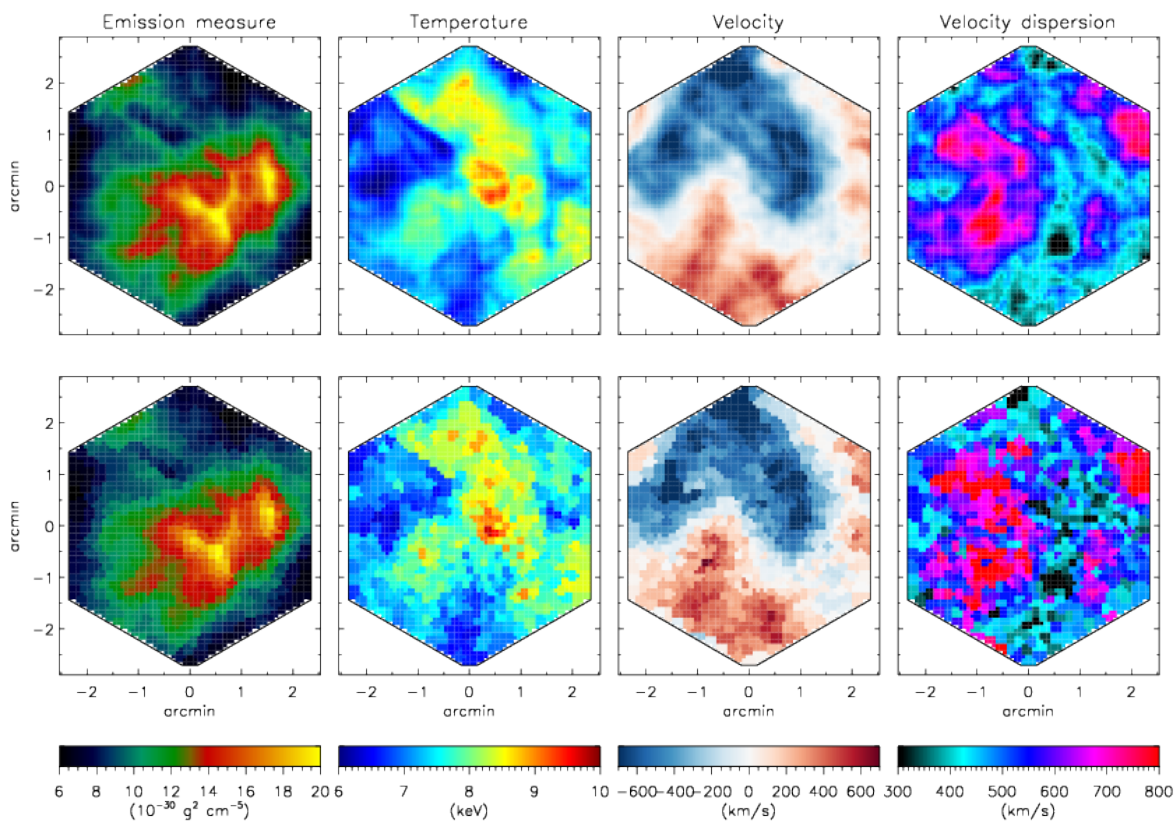

Fig. 15 True (top) and reconstructed (bottom) images of gas emissivity (far left), temperature (center-left), bulk velocity (center-right), and velocity dispersion (far right) for a fiducial Coma-like simulated cluster (Gaspari and Churazov 2013) observed with the Athena/X-IFU. Adapted from Roncarelli et al. (2018).

To test the capabilities of Athena/X-IFU at recovering gas motions in galaxy clusters, Roncarelli et al. (2018) presented mock X-IFU observations of a Coma-like cluster simulated at high resolution with the hydrodynamical code FLASH4 (see Gaspari and Churazov 2013, for details). Three-dimensional data cubes extracted from the simulation were then fed into the SIXTE observation simulator (Wilms et al. 2014) to create a long (1 Ms) mock observation 
of the fiducial cluster placed at $z=0.1$, including instrumental effects such as instrument response, point-spread function, and particle background. The mock observation was then analyzed by fitting $\sim 600$ spectra binned to reach a target of 30,000 counts per region, and maps of the main quantities of interest (emission measure, temperature, bulk velocity, and velocity dispersion) were reconstructed.

In Fig. 15 (from Roncarelli et al. 2018), the reconstructed maps (bottom panels) compared to the true, input maps (top panels) are shown. The remarkable ability of X-IFU to reconstruct accurately the properties of the simulated cluster is evident. Roncarelli et al. (2018) further constrained the statistical accuracy and bias, if any, in the reconstructed measurements. In particular, the true bulk velocity of the simulated cluster can be reconstructed with a very high level of accuracy. The reconstructed velocity correlates with the true one with a Spearman correlation coefficient $r_{s}=0.960$. A clear correlation between reconstructed and true values is also found for the velocity dispersion $\left(r_{s}=0.765\right)$, although the statistical uncertainties are larger. In both cases, biases in the reconstructed values were found to be at the level of $5 \%$ or less. Overall, the simulations presented in Roncarelli et al. (2018) demonstrate that Athena/X-IFU will allow us to perform accurate and spatially-resolved observations of gas motions in the ICM, thus enabling a leap forward in our understanding of these phenomena.

The most direct estimate of the ICM viscosity would require a measurement of the dissipation scale of turbulence from the power spectrum of measured line shifts. This scale is of the order of $\sim 1-10 \mathrm{kpc}$, for reasonable estimates of the ICM turbulent properties and the assumption that viscosity arises from ion collisions. Athena's 5 arcsec resolution may reach for the first time the dissipation scale of turbulence, thus placing direct constraints on the ICM viscosity. However, if this viscosity is very low, a definitive measurement may require the $\sim 1$ " angular resolution of Lynx, a NASA large class X-ray mission concept being considered for the 2020 decadal survey (Gaskin et al. 2017).

\subsection{Synergies beyond the X-ray band}

Complementary to X-ray studies, subarcminute resolution multi-frequency SZ experiments such as (but not limited to) upgrades to ALMA, MUSTANG-2, NIKA2, and CONCERTO will greatly enhance our capability to measure the product of velocity and electron opacity of the cluster gas through the kinetic SZ effect. For further details, we refer the reader to the dedicated review on 'Astrophysics with the Spatially \& Spectrally Resolved Sunyaev-Zeldovich Effect' in this volume (Mroczkowski et al. 2018). Comparisons between observations of bulk velocities of the gas from X-ray and high-resolution kinematic SZ measurements and galaxy velocities in the optical and infrared could potentially probe the collisionality of dark matter, assuming the galaxies serve as a reliable tracer of the kinematics of the latter. Leveraging the high spectral resolution of current/next-generation radio and optical/IR telescopes (e.g., 
ALMA, MUSE, JWST, SKA, ELT) will be key to unveil the kinematics of the ensemble warm filaments and cold clouds, and perform detailed comparisons between the velocity structure of this colder phase and that of the ICM/IGrM in cool-core systems.

Synergy with observations in other wavebands is also very promising for major mergers between clusters of galaxies. Strong gas turbulence is believed to be the driver for radio halos, produced by the reacceleration of cosmic-ray electrons in the cluster magnetic field (see related chapter by van Weeren et al. 2019 in this volume). X-ray measurements of line broadening in clusters, in combination with powerful new radio data obtained from LOFAR and the JVLA, will determine if the level of turbulence in the ICM is strong enough to produce the observed radio emission.

Acknowledgements A.S. gratefully acknowledges support by the Women In Science Excel (WISE) programme of the Netherlands Organisation for Scientific Research (NWO). M.G. is supported by NASA through Einstein Postdoctoral Fellowship Award Number PF5-160137 issued by the Chandra X-ray Observatory Center, which is operated by the SAO for and on behalf of NASA under contract NAS8-03060. Support for this work was also provided by Chandra grant GO7-18121X. D.N. acknowledges Yale University for granting a triennial leave and the Max-Planck-Institut für Astrophysik for hospitality when this work was carried out. N.W. is supported by the Lendület LP2016-11 grant awarded by the Hungarian Academy of Sciences. E.R. acknowledges the support of STFC, through the University of Hulls Consolidated Grant ST/R000840/1 and access to viper, the University of Hull High Performance Computing Facility.

\section{References}

Z. Abdulla, J.E. Carlstrom, A.B. Mantz, D.P. Marrone, C.H. Greer, et al., Constraints on the Thermal Contents of the X-ray Cavities of Cluster MS 0735.6+7421 with SunyaevZel'dovich Effect Observations. arXiv e-prints 1806.05050 (2018)

M. Ackermann, M. Ajello, A. Allafort, L. Baldini, J. Ballet, et al., GeV Gammaray Flux Upper Limits from Clusters of Galaxies. ApJ 717, 71-78 (2010). https://doi.org/10.1088/2041-8205/717/1/L71

M. Ackermann, M. Ajello, A. Albert, A. Allafort, W.B. Atwood, et al., Search for Cosmic-Ray-induced Gamma-Ray Emission in Galaxy Clusters. ApJ 787, 18 (2014). https://doi.org/10.1088/0004-637X/787/1/18

R. Adam, I. Bartalucci, G.W. Pratt, P. Ade, P. André, et al., Mapping the kinetic SunyaevZel'dovich effect toward MACS J0717.5+3745 with NIKA. A\&A 598, 115 (2017). https://doi.org/10.1051/0004-6361/201629182

F. Akimoto, A. Furuzawa, Y. Tawara, K. Yamashita, Iron K-Line Analysis of Clusters of Galaxies with the Resonance Scattering Effect. Advances in Space Research 25, 603-606 (2000). https://doi.org/10.1016/S0273-1177(99)00809-1

M.E. Anderson, R. Sunyaev, Searching for FUV line emission from $10^{7} \mathrm{~K}$ gas in massive elliptical galaxies and galaxy clusters as a tracer of turbulent velocities. MNRAS 459 2806-2821 (2016). https://doi.org/10.1093/mnras/stw822

M.E. Anderson, R. Sunyaev, FUV line emission, gas kinematics, and discovery of $[\mathrm{Fe}$ XXI] $\lambda 1354.1$ in the sightline toward a filament in M87. A\&A 617, 123 (2018). https://doi.org/10.1051/0004-6361/201732510

K. Anton, S. Wagner, I. Appenzeller, Detection of the coronal forbidden line Fe X 6374 A in the cooling flow cluster A 1795. A\&A 246, 51-54 (1991) 
P. Arévalo, E. Churazov, I. Zhuravleva, W.R. Forman, C. Jones, On the Nature of X-ray Surface Brightness Fluctuations in M87. ApJ 818, 14 (2016). https://doi.org/10.3847/0004$637 \mathrm{X} / 818 / 1 / 14$

Y. Ascasibar, M. Markevitch, The Origin of Cold Fronts in the Cores of Relaxed Galaxy Clusters. ApJ 650, 102-127 (2006). https://doi.org/10.1086/506508

S.A. Balbus, Stability, Instability, and "Backward" Transport in Stratified Fluids. ApJ 534, 420-427 (2000). https://doi.org/10.1086/308732

S.A. Balbus, N. Soker, Theory of local thermal instability in spherical systems. ApJ 341, 611-630 (1989). https://doi.org/10.1086/167521

S.A. Balbus, N. Soker, Resonant excitation of internal gravity waves in cluster cooling flows. ApJ 357, 353-366 (1990). https://doi.org/10.1086/168926

C.J. Bambic, B.J. Morsony, C.S. Reynolds, Suppression of AGN-driven Turbulence by Magnetic Fields in a Magnetohydrodynamic Model of the Intracluster Medium. ApJ 857, 84 (2018). https://doi.org/10.3847/1538-4357/aab558

P. Barai, G. Murante, S. Borgani, M. Gaspari, G.L. Granato, et al., Kinetic AGN feedback effects on cluster cool cores simulated using SPH. MNRAS 461, 1548-1567 (2016). https://doi.org/10.1093/mnras/stw1389

D. Barret, T. Lam Trong, J.-W. den Herder, L. Piro, M. Cappi, et al., The ATHENA X-ray Integral Field Unit (X-IFU), in Space Telescopes and Instrumentation 2018: Ultraviolet to Gamma Ray. Society of Photo-Optical Instrumentation Engineers (SPIE) Conference Series, vol. 10699, 2018, p. 106991. https://doi.org/10.1117/12.2312409

N. Battaglia, J.R. Bond, C. Pfrommer, J.L. Sievers, On the Cluster Physics of Sunyaev-Zel'dovich and X-Ray Surveys. I. The Influence of Feedback, Non-thermal Pressure, and Cluster Shapes on Y-M Scaling Relations. ApJ 758, 74 (2012). https://doi.org/10.1088/0004-637X/758/2/74

M.C. Begelman, Impact of Active Galactic Nuclei on the Surrounding Medium, in Gas and Galaxy Evolution, ed. by J.E. Hibbard, M. Rupen, J.H. van Gorkom Astronomical Society of the Pacific Conference Series, vol. 240, 2001, p. 363

V. Biffi, S. Borgani, G. Murante, E. Rasia, S. Planelles, et al., On the Nature of Hydrostatic Equilibrium in Galaxy Clusters. ApJ 827, 112 (2016). https://doi.org/10.3847/0004$637 \mathrm{X} / 827 / 2 / 112$

M. Birkinshaw, The Sunyaev-Zel'dovich effect. Phys. Rep. 310, 97-195 (1999). https://doi.org/10.1016/S0370-1573(98)00080-5

L. Bîrzan, D.A. Rafferty, B.R. McNamara, M.W. Wise, P.E.J. Nulsen, A Systematic Study of Radio-induced X-Ray Cavities in Clusters, Groups, and Galaxies. ApJ 607, 800-809 (2004). https://doi.org/10.1086/383519

E.L. Blanton, S.W. Randall, T.E. Clarke, C.L. Sarazin, B.R. McNamara, et al., A Very Deep Chandra Observation of A2052: Bubbles, Shocks, and Sloshing. ApJ 737, 99 (2011). https://doi.org/10.1088/0004-637X/737/2/99

H. Boehringer, G.E. Morfill, On the dynamical role of cosmic rays in cooling flows in clusters of galaxies. ApJ 330, 609-619 (1988). https://doi.org/10.1086/166497

H. Böhringer, W. Voges, A.C. Fabian, A.C. Edge, D.M. Neumann, A ROSAT HRI study of the interaction of the X-ray-emitting gas and radio lobes of NGC 1275. MNRAS 264 25-28 (1993). https://doi.org/10.1093/mnras/264.1.L25

A. Bonafede, M. Brüggen, D. Rafferty, I. Zhuravleva, C.J. Riseley, et al., LOFAR discovery of radio emission in MACS J0717.5+3745. MNRAS 478, 2927-2938 (2018). https://doi.org/10.1093/mnras/sty1121

M.A. Bourne, D. Sijacki, AGN jet feedback on a moving mesh: cocoon inflation, gas flows and turbulence. MNRAS 472, 4707-4735 (2017). https://doi.org/10.1093/mnras/stx2269

J.N. Bregman, A.C. Fabian, E.D. Miller, J.A. Irwin, On VI Observations of Galaxy Clusters: Evidence for Modest Cooling Flows. ApJ 642, 746-751 (2006) https://doi.org/10.1086/501112

M. Brüggen, C.R. Kaiser, Hot bubbles from active galactic nuclei as a heat source in coolingflow clusters. Nature 418, 301-303 (2002). https://doi.org/10.1038/nature00857

G.E. Bulbul, R.K. Smith, A. Foster, J. Cottam, M. Loewenstein, et al., High-resolution XMM-Newton Spectroscopy of the Cooling Flow Cluster A3112. ApJ 747, 32 (2012). https://doi.org/10.1088/0004-637X/747/1/32

R.E.A. Canning, A.C. Fabian, R.M. Johnstone, J.S. Sanders, C.S. Crawford, et al., Detection 
of optical coronal emission from $10^{6}-\mathrm{K}$ gas in the core of the Centaurus cluster. MNRAS 411, 411-421 (2011). https://doi.org/10.1111/j.1365-2966.2010.17693.x

M. Chatzikos, R.J.R. Williams, G.J. Ferland, R.E.A. Canning, A.C. Fabian, et al., Implications of coronal line emission in NGC 4696*. MNRAS 446, 1234-1244 (2015). https://doi.org/10.1093/mnras/stu2173

E. Churazov, W. Forman, C. Jones, H. Böhringer, Asymmetric, arc minute scale structures around NGC 1275. A\&A 356, 788-794 (2000)

E. Churazov, M. Brüggen, C.R. Kaiser, H. Böhringer, W. Forman, Evolution of Buoyant Bubbles in M87. ApJ 554, 261-273 (2001). https://doi.org/10.1086/321357

E. Churazov, R. Sunyaev, W. Forman, H. Böhringer, Cooling flows as a calorimeter of active galactic nucleus mechanical power. MNRAS 332, 729-734 (2002). https://doi.org/10.1046/j.1365-8711.2002.05332.x

E. Churazov, W. Forman, C. Jones, H. Böhringer, XMM-Newton Observations of the Perseus Cluster. I. The Temperature and Surface Brightness Structure. ApJ 590, 225-237 (2003). https://doi.org/10.1086/374923

E. Churazov, W. Forman, C. Jones, R. Sunyaev, H. Böhringer, XMM-Newton observations of the Perseus cluster - II. Evidence for gas motions in the core. MNRAS 347, 29-35 (2004). https://doi.org/10.1111/j.1365-2966.2004.07201.x

E. Churazov, I. Zhuravleva, S. Sazonov, R. Sunyaev, Resonant Scattering of X-ray Emission Lines in the Hot Intergalactic Medium. Space Sci. Rev. 157, 193-209 (2010). https://doi.org/10.1007/s11214-010-9685-4

E. Churazov, A. Vikhlinin, I. Zhuravleva, A. Schekochihin, I. Parrish, et al., X-ray surface brightness and gas density fluctuations in the Coma cluster. MNRAS 421, 1123-1135 (2012). https://doi.org/10.1111/j.1365-2966.2011.20372.x

E. Churazov, P. Arevalo, W. Forman, C. Jones, A. Schekochihin, et al., Arithmetic with $\mathrm{X}$-ray images of galaxy clusters: effective equation of state for small-scale perturbations in the ICM. MNRAS 463, 1057-1067 (2016). https://doi.org/10.1093/mnras/stw2044

L. Ciotti, J.P. Ostriker, Cooling Flows and Quasars. II. Detailed Models of Feedbackmodulated Accretion Flows. ApJ 551, 131-152 (2001). https://doi.org/10.1086/320053

L.L. Cowie, Theoretical models of X-ray emission from rich clusters of galaxies, in X-ray Astronomy with the Einstein Satellite, ed. by R. Giacconi Astrophysics and Space Science Library, vol. 87, 1981, pp. 227-240. https://doi.org/10.1007/978-94-009-8459-2_13

C.S. Crawford, S.W. Allen, H. Ebeling, A.C. Edge, A.C. Fabian, The ROSAT Brightest Cluster Sample - III. Optical spectra of the central cluster galaxies. MNRAS 306, $857-$ 896 (1999). https://doi.org/10.1046/j.1365-8711.1999.02583.x

L.P. David, J. Lim, W. Forman, J. Vrtilek, F. Combes, et al., Molecular Gas in the X-Ray Bright Group NGC 5044 as Revealed by ALMA. ApJ 792, 94 (2014). https://doi.org/10.1088/0004-637X/792/2/94

S. De Grandi, D. Eckert, S. Molendi, M. Girardi, E. Roediger, et al., A textbook example of ram-pressure stripping in the Hydra A/A780 cluster. A\&A 592, 154 (2016). https://doi.org/10.1051/0004-6361/201526641

J. de Plaa, I. Zhuravleva, N. Werner, J.S. Kaastra, E. Churazov, et al., Estimating turbulent velocities in the elliptical galaxies NGC 5044 and NGC 5813. A\&A 539, 34 (2012). https://doi.org/10.1051/0004-6361/201118404

J. de Plaa, J.S. Kaastra, N. Werner, C. Pinto, P. Kosec, et al., CHEERS: The chemical evolution RGS sample. A\&A 607, 98 (2017). https://doi.org/10.1051/0004-6361/201629926

K. Dolag, F. Vazza, G. Brunetti, G. Tormen, Turbulent gas motions in galaxy cluster simulations: the role of smoothed particle hydrodynamics viscosity. MNRAS 364, 753-772 (2005)

M. Donahue, J.T. Stocke, Coronal line emission in cluster cooling flows. ApJ 422, 459-466 (1994). https://doi.org/10.1086/173741

J. Donnert, F. Vazza, M. Brüggen, J. ZuHone, Magnetic Field Amplification in Galaxy Clusters and Its Simulation. Space Sci. Rev. 214, 122 (2018). https://doi.org/10.1007/s11214-018-0556-8

R. Dupke, R.E. White III, J.N. Bregman, Different Methods of Forming Cold Fronts in Nonmerging Clusters. ApJ 671, 181-189 (2007b). https://doi.org/10.1086/522194

R.A. Dupke, J.N. Bregman, Detection of Bulk Motions in the Intracluster Medium of the Centaurus Cluster. ApJ 562, 266-278 (2001a). https://doi.org/10.1086/323433 
R.A. Dupke, J.N. Bregman, Velocity Gradients in the Intracluster Gas of the Perseus Cluster. ApJ 547, 705-713 (2001b). https://doi.org/10.1086/318393

R.A. Dupke, J.N. Bregman, Direct Measurements of Gas Bulk Flows in the Intracluster Medium of the Centaurus Cluster with the Chandra Satellite. ApJ 639, 781-787 (2006). https://doi.org/10.1086/499343

R.A. Dupke, N. Mirabal, J.N. Bregman, A.E. Evrard, The Merger in Abell 576: A Line-ofSight Bullet Cluster? ApJ 668, 781-795 (2007a). https://doi.org/10.1086/520708

D. Eckert, S. Molendi, M. Owers, M. Gaspari, T. Venturi, et al., The stripping of a galaxy group diving into the massive cluster A2142. A\&A 570, 119 (2014). https://doi.org/10.1051/0004-6361/201424259

D. Eckert, M. Gaspari, F. Vazza, F. Gastaldello, A. Tramacere, et al., On the Connection between Turbulent Motions and Particle Acceleration in Galaxy Clusters. ApJ 843, 29 (2017a). https://doi.org/10.3847/2041-8213/aa7c1a

D. Eckert, M. Gaspari, M.S. Owers, E. Roediger, S. Molendi, et al., Deep Chandra observations of the stripped galaxy group falling into Abell 2142. A\&A 605, 25 (2017b). https://doi.org/10.1051/0004-6361/201730555

A.C. Edge, The detection of molecular gas in the central galaxies of cooling flow clusters. MNRAS 328, 762-782 (2001). https://doi.org/10.1046/j.1365-8711.2001.04802.x

S. Ehlert, N. Werner, A. Simionescu, S.W. Allen, J.D.P. Kenney, et al., Ripping apart at the seams: the network of stripped gas surrounding M86. MNRAS 430(3), 2401-2410 (2013). https://doi.org/10.1093/mnras/stt060

S. Ettori, G.W. Pratt, J. de Plaa, D. Eckert, J. Nevalainen, et al., The Hot and Energetic Universe: The astrophysics of galaxy groups and clusters. arXiv e-prints 1306.2322 (2013)

A.C. Fabian, Cooling Flows in Clusters of Galaxies. ARA\&A 32, 277-318 (1994). https://doi.org/10.1146/annurev.aa.32.090194.001425

A.C. Fabian, Observational Evidence of Active Galactic Nuclei Feedback. ARA\&A 50, 455489 (2012). https://doi.org/10.1146/annurev-astro-081811-125521

A.C. Fabian, P.E.J. Nulsen, Subsonic accretion of cooling gas in clusters of galaxies. MNRAS 180, 479-484 (1977). https://doi.org/10.1093/mnras/180.3.479

A.C. Fabian, J.S. Sanders, S.W. Allen, C.S. Crawford, K. Iwasawa, et al., A deep Chandra observation of the Perseus cluster: shocks and ripples. MNRAS 344, 43-47 (2003a). https://doi.org/10.1046/j.1365-8711.2003.06902.x

A.C. Fabian, J.S. Sanders, C.S. Crawford, C.J. Conselice, J.S. Gallagher, R.F.G. Wyse, The relationship between the optical $\mathrm{H} \alpha$ filaments and the X-ray emission in the core of the Perseus cluster. MNRAS 344, 48-52 (2003b). https://doi.org/10.1046/j.13658711.2003.06856.x

A.C. Fabian, J.S. Sanders, G.B. Taylor, S.W. Allen, C.S. Crawford, et al., A very deep Chandra observation of the Perseus cluster: shocks, ripples and conduction. MNRAS 366, 417-428 (2006). https://doi.org/10.1111/j.1365-2966.2005.09896.x

A.C. Fabian, J.S. Sanders, S.W. Allen, R.E.A. Canning, E. Churazov, et al., A wide Chandra view of the core of the Perseus cluster. MNRAS 418, 2154-2164 (2011). https://doi.org/10.1111/j.1365-2966.2011.19402.x

A.C. Fabian, S.A. Walker, H.R. Russell, C. Pinto, J.S. Sanders, C.S. Reynolds, Do sound waves transport the AGN energy in the Perseus cluster? MNRAS 464, 1-5 (2017). https://doi.org/10.1093/mnrasl/slw170

A. Finoguenov, H. Böhringer, Y.-Y. Zhang, XMM-Newton study of the two-dimensional structure of the REFLEX-DXL galaxy clusters. A\&A 442, 827-839 (2005). https://doi.org/10.1051/0004-6361:20053306

W. Forman, C. Jones, E. Churazov, M. Markevitch, P. Nulsen, et al., Filaments, Bubbles, and Weak Shocks in the Gaseous Atmosphere of M87. ApJ 665, 1057-1066 (2007). https://doi.org/10.1086/519480

W. Forman, E. Churazov, C. Jones, S. Heinz, R. Kraft, A. Vikhlinin, Partitioning the Outburst Energy of a Low Eddington Accretion Rate AGN at the Center of an Elliptical Galaxy: The Recent 12 Myr History of the Supermassive Black Hole in M87. ApJ 844, 122 (2017). https://doi.org/10.3847/1538-4357/aa70e4

Y. Fujita, T.H. Reiprich, Can Supermassive Black Holes Sufficiently Heat Cool Cores of Galaxy Clusters? ApJ 612, 797-804 (2004). https://doi.org/10.1086/422800 
J.A. Gaskin, R. Allured, S.R. Bandler, S. Basso, M.W. Bautz, et al., Lynx Mission concept status, in Society of Photo-Optical Instrumentation Engineers (SPIE) Conference Series. Society of Photo-Optical Instrumentation Engineers (SPIE) Conference Series, vol. 10397, 2017, p. 103970. https://doi.org/10.1117/12.2273911

M. Gaspari, E. Churazov, Constraining turbulence and conduction in the hot ICM through density perturbations. A\&A 559, 78 (2013). https://doi.org/10.1051/0004$6361 / 201322295$

M. Gaspari, A. Sądowski, Unifying the Micro and Macro Properties of AGN Feeding and Feedback. ApJ 837, 149 (2017). https://doi.org/10.3847/1538-4357/aa61a3

M. Gaspari, F. Brighenti, M. Ruszkowski, Solving the cooling flow problem through mechanical AGN feedback. Astronomische Nachrichten 334, 394 (2013b). https://doi.org/10.1002/asna.201211865

M. Gaspari, F. Brighenti, P. Temi, Mechanical AGN feedback: controlling the thermodynamical evolution of elliptical galaxies. MNRAS 424, 190-209 (2012b). https://doi.org/10.1111/j.1365-2966.2012.21183.x

M. Gaspari, F. Brighenti, P. Temi, Chaotic cold accretion on to black holes in rotating atmospheres. A\&A 579, 62 (2015). https://doi.org/10.1051/0004-6361/201526151

M. Gaspari, M. Ruszkowski, S.P. Oh, Chaotic cold accretion on to black holes. MNRAS 432, 3401-3422 (2013a). https://doi.org/10.1093/mnras/stt692

M. Gaspari, M. Ruszkowski, P. Sharma, Cause and Effect of Feedback: Multiphase Gas in Cluster Cores Heated by AGN Jets. ApJ 746, 94 (2012a). https://doi.org/10.1088/0004$637 \mathrm{X} / 746 / 1 / 94$

M. Gaspari, P. Temi, F. Brighenti, Raining on black holes and massive galaxies: the top-down multiphase condensation model. MNRAS 466, 677-704 (2017). https://doi.org/10.1093/mnras/stw3108

M. Gaspari, C. Melioli, F. Brighenti, A. D'Ercole, The dance of heating and cooling in galaxy clusters: three-dimensional simulations of self-regulated active galactic nuclei outflows. MNRAS 411, 349-372 (2011). https://doi.org/10.1111/j.1365-2966.2010.17688.x

M. Gaspari, E. Churazov, D. Nagai, E.T. Lau, I. Zhuravleva, The relation between gas density and velocity power spectra in galaxy clusters: High-resolution hydrodynamic simulations and the role of conduction. A\&A 569, 67 (2014). https://doi.org/10.1051/0004$6361 / 201424043$

M. Gaspari, M. McDonald, S.L. Hamer, F. Brighenti, P. Temi, et al., Shaken Snow Globes: Kinematic Tracers of the Multiphase Condensation Cascade in Massive Galaxies, Groups, and Clusters. ApJ 854, 167 (2018). https://doi.org/10.3847/1538-4357/aaaa1b

F. Gastaldello, S. Molendi, Ni Abundance in the Core of the Perseus Cluster: An Answer to the Significance of Resonant Scattering. ApJ 600, 670-680 (2004). https://doi.org/10.1086/379970

M. Gendron-Marsolais, J. Hlavacek-Larrondo, T.B. Martin, L. Drissen, M. McDonald, et al., Revealing the velocity structure of the filamentary nebula in NGC 1275 in its entirety. MNRAS 479, 28-33 (2018). https://doi.org/10.1093/mnrasl/sly084

M.R. Gilfanov, R.A. Sunyaev, E.M. Churazov, Radial Brightness Profiles of Resonance XRay Lines in Galaxy Clusters. Soviet Astronomy Letters 13, 3-7 (1987)

M. Gitti, P.E.J. Nulsen, L.P. David, B.R. McNamara, M.W. Wise, A Chandra Study of the Large-scale Shock and Cool Filaments in Hydra A: Evidence for Substantial Gas Dredge-up by the Central Outburst. ApJ 732, 13 (2011). https://doi.org/10.1088/0004$637 \mathrm{X} / 732 / 1 / 13$

C.M. Graney, C.L. Sarazin, Optical coronal emission lines from equilibrium and cooling plasmas. ApJ 364, 561-567 (1990). https://doi.org/10.1086/169438

L. Gu, H. Xu, J. Gu, Y. Wang, Z. Zhang, et al., A Chandra Study of Temperature Substructures in Intermediate-Redshift Galaxy Clusters. ApJ 700, 1161-1172 (2009). https://doi.org/10.1088/0004-637X/700/2/1161

L. Gu, I. Zhuravleva, E. Churazov, F. Paerels, J. Kaastra, H. Yamaguchi, X-Ray Spectroscopy of Galaxy Clusters: Beyond the CIE Modeling. Space Sci. Rev. 214, 108 (2018). https://doi.org/10.1007/s11214-018-0544-z

S.F. Gull, K.J.E. Northover, Bubble Model of Extragalactic Radio Sources. Nature 244, 80-83 (1973). https://doi.org/10.1038/244080a0

E.J. Hallman, M. Markevitch, Chandra Observation of the Merging Cluster A168: 
A Late Stage in the Evolution of a Cold Front. ApJ 610, 81-84 (2004). https://doi.org/10.1086/423449

S.L. Hamer, A.C. Edge, A.M. Swinbank, R.J. Wilman, F. Combes, et al., Optical emission line nebulae in galaxy cluster cores 1: the morphological, kinematic and spectral properties of the sample. MNRAS 460, 1758-1789 (2016). https://doi.org/10.1093/mnras/stw1054

N.A. Hatch, C.S. Crawford, A.C. Fabian, R.M. Johnstone, Detections of molecular hydrogen in the outer filaments of NGC1275. MNRAS 358, 765-773 (2005). https://doi.org/10.1111/j.1365-2966.2005.08787.x

T.M. Heckman, S.A. Baum, W.J.M. van Breugel, P. McCarthy, Dynamical, physical, and chemical properties of emission-line nebulae in cooling flows. ApJ 338, 48-77 (1989). https://doi.org/10.1086/167181

S. Heinz, M. Brüggen, B. Morsony, Prospects of High-Resolution X-ray Spectroscopy for Active Galactic Nucleus Feedback in Galaxy Clusters. ApJ 708, 462-468 (2010). https://doi.org/10.1088/0004-637X/708/1/462

S. Heinz, C.S. Reynolds, M.C. Begelman, X-Ray Signatures of Evolving Radio Galaxies. ApJ 501, 126-136 (1998). https://doi.org/10.1086/305807

Hitomi Collaboration, The quiescent intracluster medium in the core of the Perseus cluster Nature 535, 117-121 (2016). https://doi.org/10.1038/nature18627

Hitomi Collaboration, Atmospheric gas dynamics in the Perseus cluster observed with Hitomi. PASJ 70, 9 (2018a). https://doi.org/10.1093/pasj/psx138

Hitomi Collaboration, Measurements of resonant scattering in the Perseus Cluster core with Hitomi SXS. PASJ 70, 10 (2018b). https://doi.org/10.1093/pasj/psx127

J. Hlavacek-Larrondo, A.C. Fabian, A.C. Edge, H. Ebeling, J.S. Sanders, et al., Extreme AGN feedback in the MAssive Cluster Survey: a detailed study of X-ray cavities at $\mathrm{z}>0.3$ MNRAS 421, 1360-1384 (2012). https://doi.org/10.1111/j.1365-2966.2011.20405.x

F. Hofmann, J.S. Sanders, K. Nandra, N. Clerc, M. Gaspari, Thermodynamic perturbations in the X-ray halo of 33 clusters of galaxies observed with Chandra ACIS. A\&A 585 130 (2016). https://doi.org/10.1051/0004-6361/201526925

E.M. Hu, L.L. Cowie, Z. Wang, Long-slit spectroscopy of gas in the cores of X-ray luminous clusters. ApJS 59, 447-498 (1985). https://doi.org/10.1086/191081

B. Huber, C. Tchernin, D. Eckert, C. Farnier, A. Manalaysay, et al., Probing the cosmic-ray content of galaxy clusters by stacking Fermi-LAT count maps. A\&A 560, 64 (2013) https://doi.org/10.1051/0004-6361/201321947

L. Iapichino, W. Schmidt, J.C. Niemeyer, J. Merklein, Turbulence production and turbulent pressure support in the intergalactic medium. MNRAS 414, 2297-2308 (2011). https://doi.org/10.1111/j.1365-2966.2011.18550.x

Y. Ichinohe, N. Werner, A. Simionescu, S.W. Allen, R.E.A. Canning, et al., The growth of the galaxy cluster Abell 85: mergers, shocks, stripping and seeding of clumping. MNRAS 448, 2971-2986 (2015). https://doi.org/10.1093/mnras/stv217

Y. Ichinohe, A. Simionescu, N. Werner, T. Takahashi, An azimuthally resolved study of the cold front in Abell 3667. MNRAS 467, 3662-3676 (2017). https://doi.org/10.1093/mnras/stx280

Y. Ichinohe, A. Simionescu, N. Werner, A.C. Fabian, T. Takahashi, Substructures associated with the sloshing cold front in the Perseus cluster. MNRAS 483, 1744-1753 (2019). https://doi.org/10.1093/mnras/sty3257

N.A. Inogamov, R.A. Sunyaev, Turbulence in Clusters of Galaxies and X-ray Line Profiles. Astronomy Letters 29, 791-824 (2003)

J.S. Kaastra, R. Lieu, J.P.D. Mittaz, J.A.M. Bleeker, R. Mewe, et al., High- and Low-Energy Nonthermal X-Ray Emission from the Abell 2199 Cluster of Galaxies. ApJ 519, 119-122 (1999). https://doi.org/10.1086/312112

S.M. Kahn, J.R. Peterson, F.B.S. Paerels, H. Xu, J.S. Kaastra, et al., High Resolution X-Ray Spectroscopic Constraints on Cooling Flow Models of Clusters of Galaxies and Gaseous Haloes Around Elliptical Galaxies, in Matter and Energy in Clusters of Galaxies, ed. by S. Bowyer, C.-Y. Hwang Astronomical Society of the Pacific Conference Series, vol. 301, 2003, p. 23

H. Kawahara, E.D. Reese, T. Kitayama, S. Sasaki, Y. Suto, Extracting Galaxy Cluster Gas Inhomogeneity from X-Ray Surface Brightness: A Statistical Approach and Application 
to Abell 3667. ApJ 687, 936-950 (2008). https://doi.org/10.1086/591930

R. Khatri, M. Gaspari, Thermal SZ fluctuations in the ICM: probing turbulence and thermodynamics in Coma cluster with Planck. MNRAS 463, 655-669 (2016). https://doi.org/10.1093/mnras/stw2027

C.C. Kirkpatrick, B.R. McNamara, Hot outflows in galaxy clusters. MNRAS 452, 4361-4376 (2015). https://doi.org/10.1093/mnras/stv1574

C.C. Kirkpatrick, B.R. McNamara, K.W. Cavagnolo, Anisotropic Metal-enriched Outflows Driven by Active Galactic Nuclei in Clusters of Galaxies. ApJ 731, 23 (2011). https://doi.org/10.1088/2041-8205/731/2/L23

C.C. Kirkpatrick, M. Gitti, K.W. Cavagnolo, B.R. McNamara, L.P. David, et al., Direct Evidence for Outflow of Metal-Enriched Gas Along the Radio Jets of Hydra A. ApJ 707, 69-72 (2009). https://doi.org/10.1088/0004-637X/707/1/L69

T. Kitayama, M. Bautz, M. Markevitch, K. Matsushita, S. Allen, et al., ASTRO-H White Paper - Clusters of Galaxies and Related Science. arXiv e-prints 1412.1176 (2014)

J. Kormendy, L.C. Ho, Coevolution (Or Not) of Supermassive Black Holes and Host Galaxies. ARA\&A 51, 511-653 (2013). https://doi.org/10.1146/annurev-astro-082708-101811

R.P. Kraft, W.R. Forman, C. Jones, P.E.J. Nulsen, M.J. Hardcastle, et al., THE GAS DYNAMICS OF NGC 4472 REVEALED BY XMM-NEWTON. ApJ 727(1), 41 (2011). https://doi.org/10.1088/0004-637X/727/1/41

R. Kraft, E. Roediger, M. Machacek, W.R. Forman, P.E.J. Nulsen, et al., Stripped elliptical galaxies as probes of ICM physics: III. Deep Chandra Observation of NGC 4552 - Measuring the Viscosity of the Intracluster Medium. ApJ 848, 27 (2017). https://doi.org/10.3847/1538-4357/aa8a6e

A.V. Kravtsov, S. Borgani, Formation of Galaxy Clusters. ARA\&A 50, 353-409 (2012). https://doi.org/10.1146/annurev-astro-081811-125502

M.W. Kunz, A.A. Schekochihin, S.C. Cowley, J.J. Binney, J.S. Sanders, A thermally stable heating mechanism for the intracluster medium: turbulence, magnetic fields and plasma instabilities. MNRAS 410, 2446-2457 (2011). https://doi.org/10.1111/j.13652966.2010.17621.x

E.T. Lau, A.V. Kravtsov, D. Nagai, Residual Gas Motions in the Intracluster Medium and Bias in Hydrostatic Measurements of Mass Profiles of Clusters. ApJ 705, 1129-1138 (2009). https://doi.org/10.1088/0004-637X/705/2/1129

E.T. Lau, M. Gaspari, D. Nagai, P. Coppi, Physical Origins of Gas Motions in Galaxy Cluster Cores: Interpreting Hitomi Observations of the Perseus Cluster. ApJ 849, 54 (2017). https://doi.org/10.3847/1538-4357/aa8c00

Y. Li, G.L. Bryan, Modeling Active Galactic Nucleus Feedback in Cool-core Clusters: The Formation of Cold Clumps. ApJ 789, 153 (2014). https://doi.org/10.1088/0004$637 \mathrm{X} / 789 / 2 / 153$

Y. Li, M. Ruszkowski, G.L. Bryan, AGN Heating in Simulated Cool-core Clusters. ApJ 847, 106 (2017). https://doi.org/10.3847/1538-4357/aa88c1

M. Loewenstein, E.G. Zweibel, M.C. Begelman, Cosmic-ray heating of cooling flows - A critical analysis. ApJ 377, 392-402 (1991). https://doi.org/10.1086/170369

N. Lyskova, E. Churazov, C. Zhang, W. Forman, C. Jones, et al., Close-up view of an ongoing merger between the NGC 4839 group and the Coma cluster - a post-merger scenario. arXiv e-prints 1811.07944 (2018)

M. Lyutikov, Dissipation in Intercluster Plasma. ApJ 668, 1-4 (2007). https://doi.org/10.1086/522696

M. Machacek, A. Dosaj, W. Forman, C. Jones, M. Markevitch, et al., Infall of the Elliptical Galaxy NGC 1404 into the Fornax Cluster. ApJ 621, 663-672 (2005). https://doi.org/10.1086/427548

A. Malagoli, R. Rosner, G. Bodo, On the thermal instability of galactic and cluster halos. ApJ 319, 632-636 (1987). https://doi.org/10.1086/165483

M. Markevitch, A. Vikhlinin, Shocks and cold fronts in galaxy clusters. Phys. Rep. 443 1-53 (2007). https://doi.org/10.1016/j.physrep.2007.01.001

M. Markevitch, A. Vikhlinin, P. Mazzotta, Nonhydrostatic Gas in the Core of the Relaxed Galaxy Cluster A1795. ApJ 562, 153-156 (2001). https://doi.org/10.1086/337973

M. Markevitch, T.J. Ponman, P.E.J. Nulsen, M.W. Bautz, D.J. Burke, et al., Chandra Observation of Abell 2142: Survival of Dense Subcluster Cores in a Merger. ApJ 541, 
542-549 (2000). https://doi.org/10.1086/309470

M. Markevitch, A.H. Gonzalez, L. David, A. Vikhlinin, S. Murray, et al., A Textbook Example of a Bow Shock in the Merging Galaxy Cluster 1E 0657-56. ApJ 567, 27-31 (2002). https://doi.org/10.1086/339619

W.G. Mathews, J.N. Bregman, Radiative accretion flow onto giant galaxies in clusters. ApJ 224, 308-319 (1978). https://doi.org/10.1086/156379

W.G. Mathews, D.A. Buote, F. Brighenti, Spatial Diffusion of X-Ray Emission Lines in the M87 Cooling Flow; Evidence for Absorption. ApJ 550, 31-34 (2001). https://doi.org/10.1086/319497

P. Mazzotta, A.C. Edge, M. Markevitch, A Chandra Study of the Complex Structure in the Core of 2A 0335+096. ApJ 596, 190-203 (2003). https://doi.org/10.1086/377633

M. McCourt, P. Sharma, E. Quataert, I.J. Parrish, Thermal instability in gravitationally stratified plasmas: implications for multiphase structure in clusters and galaxy haloes. MNRAS 419, 3319-3337 (2012). https://doi.org/10.1111/j.1365-2966.2011.19972.x

M. McDonald, L.H. Wei, S. Veilleux, Cold Molecular Gas along the Cooling X-Ray Filament in A1795. ApJ 755, 24 (2012). https://doi.org/10.1088/2041-8205/755/2/L24

M. McDonald, S.W. Allen, M. Bayliss, B.A. Benson, L.E. Bleem, et al., The Remarkable Similarity of Massive Galaxy Clusters from $\mathrm{z} \sim 0$ to $\mathrm{z} \sim 1.9$. ApJ 843, 28 (2017). https://doi.org/10.3847/1538-4357/aa7740

B.R. McNamara, P.E.J. Nulsen, Mechanical feedback from active galactic nuclei in galaxies, groups and clusters. New Journal of Physics 14(5), 055023 (2012). https://doi.org/10.1088/1367-2630/14/5/055023

B.R. McNamara, M. Wise, P.E.J. Nulsen, L.P. David, C.L. Sarazin, et al., Chandra X-Ray Observations of the Hydra A Cluster: An Interaction between the Radio Source and the X-Ray-emitting Gas. ApJ 534, 135-138 (2000). https://doi.org/10.1086/312662

B.R. McNamara, P.E.J. Nulsen, M.W. Wise, D.A. Rafferty, C. Carilli, et al., The heating of gas in a galaxy cluster by X-ray cavities and large-scale shock fronts. Nature 433, 45-47 (2005). https://doi.org/10.1038/nature03202

B.R. McNamara, H.R. Russell, P.E.J. Nulsen, A.C. Edge, N.W. Murray, et al., A $10^{10}$ Solar Mass Flow of Molecular Gas in the A1835 Brightest Cluster Galaxy. ApJ 785, 44 (2014). https://doi.org/10.1088/0004-637X/785/1/44

B.R. McNamara, H.R. Russell, P.E.J. Nulsen, M.T. Hogan, A.C. Fabian, et al., A Mechanism for Stimulating AGN Feedback by Lifting Gas in Massive Galaxies. ApJ 830, 79 (2016). https://doi.org/10.3847/0004-637X/830/2/79

J. Merten, D. Coe, R. Dupke, R. Massey, A. Zitrin, et al., Creation of cosmic structure in the complex galaxy cluster merger Abell 2744. MNRAS 417, 333-347 (2011). https://doi.org/10.1111/j.1365-2966.2011.19266.x

F. Miniati, The Matryoshka Run: A Eulerian Refinement Strategy to Study the Statistics of Turbulence in Virialized Cosmic Structures. ApJ 782, 21 (2014). https://doi.org/10.1088/0004-637X/782/1/21

R. Mittal, C.P. O'Dea, G. Ferland, J.B.R. Oonk, A.C. Edge, et al., Herschel observations of the Centaurus cluster - the dynamics of cold gas in a cool core. MNRAS 418, 2386-2402 (2011). https://doi.org/10.1111/j.1365-2966.2011.19634.x

R. Mittal, J.B.R. Oonk, G.J. Ferland, A.C. Edge, C.P. O'Dea, et al., Herschel observations of extended atomic gas in the core of the Perseus cluster. MNRAS 426, 2957-2977 (2012). https://doi.org/10.1111/j.1365-2966.2012.21891.x

T. Mroczkowski, D. Nagai, K. Basu, J. Chluba, J. Sayers, et al., Astrophysics with the Spatially and Spectrally Resolved Sunyaev-Zeldovich Effects: A Millimetre/Submillimetre Probe of the Warm and Hot Universe. arXiv e-prints 1811.02310 (2018)

D. Nagai, A. Vikhlinin, A.V. Kravtsov, Testing X-Ray Measurements of Galaxy Clusters with Cosmological Simulations. ApJ 655, 98-108 (2007). https://doi.org/10.1086/509868

D. Nagai, E.T. Lau, C. Avestruz, K. Nelson, D.H. Rudd, Predicting Merger-induced Gas Motions in ACDM Galaxy Clusters. ApJ 777, 137 (2013). https://doi.org/10.1088/0004$637 \mathrm{X} / 777 / 2 / 137$

K. Nandra, D. Barret, X. Barcons, A. Fabian, J.-W. den Herder, et al., The Hot and Energetic Universe: A White Paper presenting the science theme motivating the Athena+ mission. arXiv e-prints 1306.2307 (2013) 
K. Nelson, E.T. Lau, D. Nagai, Hydrodynamic Simulation of Non-thermal Pressure Profiles of Galaxy Clusters. ApJ 792, 25 (2014b). https://doi.org/10.1088/0004-637X/792/1/25

K. Nelson, D.H. Rudd, L. Shaw, D. Nagai, Evolution of the Merger-induced Hydrostatic Mass Bias in Galaxy Clusters. ApJ 751, 121 (2012). https://doi.org/10.1088/0004$637 \mathrm{X} / 751 / 2 / 121$

K. Nelson, E.T. Lau, D. Nagai, D.H. Rudd, L. Yu, Weighing Galaxy Clusters with Gas. II. On the Origin of Hydrostatic Mass Bias in ACDM Galaxy Clusters. ApJ 782, 107 (2014a). https://doi.org/10.1088/0004-637X/782/2/107

D.M. Neumann, M. Arnaud, R. Gastaud, N. Aghanim, D. Lumb, et al., The NGC 4839 group falling into the Coma cluster observed by XMM-Newton. Astronomy and Astrophysics 365(1), 74-79 (2001). https://doi.org/10.1051/0004-6361:20000182

M.L. Norman, G.L. Bryan, Cluster Turbulence, in The Radio Galaxy Messier 87, ed. by H.-J. Röser, K. Meisenheimer Lecture Notes in Physics, Berlin Springer Verlag, vol. 530, 1999, p. 106. https://doi.org/10.1007/BFb0106425

A. Ogorzalek, I. Zhuravleva, S.W. Allen, C. Pinto, N. Werner, et al., Improved measurements of turbulence in the hot gaseous atmospheres of nearby giant elliptical galaxies. MNRAS 472, 1659-1676 (2017). https://doi.org/10.1093/mnras/stx2030

T.B. O'Hara, J.J. Mohr, M.A. Guerrero, A Chandra Study of the Effects of a Major Merger on the Structure of A2319. ApJ 604, 604-613 (2004). https://doi.org/10.1086/382063

H. Omma, J. Binney, Structural stability of cooling flows. MNRAS 350, 13-16 (2004). https://doi.org/10.1111/j.1365-2966.2004.07809.x

N. Ota, H. Yoshida, Search for gas bulk motions in eight nearby clusters of galaxies with Suzaku. PASJ 68, 19 (2016). https://doi.org/10.1093/pasj/psv128

N. Ota, D. Nagai, E.T. Lau, Constraining hydrostatic mass bias of galaxy clusters with high-resolution X-ray spectroscopy. PASJ $\quad \mathbf{7 0}, 51 \quad$ (2018). https://doi.org/10.1093/pasj/psy040

N. Ota, Y. Fukazawa, A.C. Fabian, T. Kanemaru, M. Kawaharada, et al., Suzaku Observations of the Centaurus Cluster: Absence of Bulk Motions in the Intracluster Medium. PASJ 59, 351-359 (2007). https://doi.org/10.1093/pasj/59.sp1.S351

M.S. Owers, S.W. Randall, P.E.J. Nulsen, W.J. Couch, L.P. David, J.C. Kempner, The Dissection of Abell 2744: A Rich Cluster Growing Through Major and Minor Mergers. ApJ 728, 27 (2011). https://doi.org/10.1088/0004-637X/728/1/27

M. Ozawa, H. Uchiyama, H. Matsumoto, H. Nakajima, K. Koyama, et al., Energy-Scale Calibration of the Suzaku X-Ray Imaging Spectrometer Using the Checker Flag Charge-Injection Technique in Orbit. PASJ 61, 1-7 (2009). https://doi.org/10.1093/pasj/61.sp1.S1

I.J. Parrish, J.M. Stone, Nonlinear Evolution of the Magnetothermal Instability in Two Dimensions. ApJ 633, 334-348 (2005). https://doi.org/10.1086/444589

I.J. Parrish, M. McCourt, E. Quataert, P. Sharma, Turbulent pressure support in the outer parts of galaxy clusters. MNRAS 419, 29-33 (2012). https://doi.org/10.1111/j.17453933.2011.01171.x

R. Paterno-Mahler, E.L. Blanton, S.W. Randall, T.E. Clarke, Deep Chandra Observations of the Extended Gas Sloshing Spiral in A2029. ApJ 773, 114 (2013). https://doi.org/10.1088/0004-637X/773/2/114

J.R. Peterson, A.C. Fabian, X-ray spectroscopy of cooling clusters. Phys. Rep. 427, 1-39 (2006). https://doi.org/10.1016/j.physrep.2005.12.007

C. Pinto, A.C. Fabian, N. Werner, P. Kosec, J. Ahoranta, et al., Discovery of O VII line emitting gas in elliptical galaxies. A\&A 572, 8 (2014). https://doi.org/10.1051/0004$6361 / 201425270$

C. Pinto, J.S. Sanders, N. Werner, J. de Plaa, A.C. Fabian, et al., Chemical Enrichment RGS cluster Sample (CHEERS): Constraints on turbulence. A\&A 575, 38 (2015). https://doi.org/10.1051/0004-6361/201425278

F. Pizzolato, N. Soker, On the Nature of Feedback Heating in Cooling Flow Clusters. ApJ 632, 821-830 (2005). https://doi.org/10.1086/444344

D. Prasad, P. Sharma, A. Babul, AGN jet-driven stochastic cold accretion in cluster cores. MNRAS 471, 1531-1542 (2017). https://doi.org/10.1093/mnras/stx1698

D.A. Prokhorov, E.M. Churazov, Counting gamma rays in the directions of galaxy clusters. A\&A 567, 93 (2014). https://doi.org/10.1051/0004-6361/201322454 
F.A. Pulido, B.R. McNamara, A.C. Edge, M.T. Hogan, A.N. Vantyghem, et al., The Origin of Molecular Clouds in Central Galaxies. ApJ 853, 177 (2018). https://doi.org/10.3847/1538-4357/aaa54b

E. Quataert, Buoyancy Instabilities in Weakly Magnetized Low-Collisionality Plasmas. ApJ 673, 758-762 (2008). https://doi.org/10.1086/525248

S.W. Randall, C. Jones, M. Markevitch, E.L. Blanton, P.E.J. Nulsen, W.R. Forman, Gas Sloshing and Bubbles in the Galaxy Group NGC 5098. ApJ 700, 1404-1414 (2009). https://doi.org/10.1088/0004-637X/700/2/1404

S.W. Randall, P.E.J. Nulsen, C. Jones, W.R. Forman, E. Bulbul, et al., A Very Deep Chandra Observation of the Galaxy Group NGC 5813: AGN Shocks, Feedback, and Outburst History. ApJ 805, 112 (2015). https://doi.org/10.1088/0004-637X/805/2/112

E. Rasia, S. Ettori, L. Moscardini, P. Mazzotta, S. Borgani, et al., Systematics in the X-ray cluster mass estimators. MNRAS 369, 2013-2024 (2006)

P. Rebusco, E. Churazov, R. Sunyaev, H. Böhringer, W. Forman, Width of X-ray lines as a diagnostic of gas motions in cooling flows. MNRAS 384, 1511-1518 (2008). https://doi.org/10.1111/j.1365-2966.2007.12770.x

Y. Revaz, F. Combes, P. Salomé, Formation of cold filaments in cooling flow clusters. A\&A 477, 33-36 (2008). https://doi.org/10.1051/0004-6361:20078915

C.S. Reynolds, S.A. Balbus, A.A. Schekochihin, Inefficient Driving of Bulk Turbulence By Active Galactic Nuclei in a Hydrodynamic Model of the Intracluster Medium. ApJ 815, 41 (2015). https://doi.org/10.1088/0004-637X/815/1/41

C.S. Reynolds, S. Heinz, M.C. Begelman, The hydrodynamics of dead radio galaxies. MNRAS 332, 271-282 (2002). https://doi.org/10.1046/j.1365-8711.2002.04724.x

E. Roediger, M. Brüggen, A. Simionescu, H. Böhringer, E. Churazov, W.R. Forman, Gas sloshing, cold front formation, and metal redistribution: the Virgo cluster as a quantitative test case. MNRAS 413, 2057-2077 (2011)

E. Roediger, R.P. Kraft, M.E. Machacek, W.R. Forman, P.E.J. Nulsen, et al., Irregular Sloshing Cold Fronts in the nearby Merging Groups NGC 7618 and UGC 12491: Evidence for Kelvin-Helmholtz Instabilities. ApJ 754, 147 (2012a). https://doi.org/10.1088/0004$637 \mathrm{X} / 754 / 2 / 147$

E. Roediger, L. Lovisari, R. Dupke, S. Ghizzardi, M. Brüggen, et al., Gas sloshing, cold fronts, Kelvin-Helmholtz instabilities and the merger history of the cluster of galaxies Abell 496. MNRAS 420, 3632-3648 (2012b). https://doi.org/10.1111/j.13652966.2011.20287.x

E. Roediger, R.P. Kraft, P. Nulsen, E. Churazov, W. Forman, et al., Viscous KelvinHelmholtz instabilities in highly ionized plasmas. MNRAS 436, 1721-1740 (2013). https://doi.org/10.1093/mnras/stt1691

E. Roediger, R.P. Kraft, P.E.J. Nulsen, W.R. Forman, M. Machacek, et al., Stripped Elliptical Galaxies as Probes of ICM Physics: I. Tails, Wakes, and Flow Patterns in and Around Stripped Ellipticals. ApJ 806, 103 (2015a). https://doi.org/10.1088/0004$637 \mathrm{X} / 806 / 1 / 103$

E. Roediger, R.P. Kraft, P.E.J. Nulsen, W.R. Forman, M. Machacek, et al., STRIPPED ELLIPTICAL GALAXIES AS PROBES OF ICM PHYSICS. II. STIRRED, BUT MIXED? VISCOUS AND INVISCID GAS STRIPPING OF THE VIRGO ELLIPTICAL M89. ApJ 806(1), 104 (2015b). https://doi.org/10.1088/0004-637X/806/1/104

M. Roncarelli, M. Gaspari, S. Ettori, V. Biffi, F. Brighenti, et al., Measuring turbulence and gas motions in galaxy clusters via synthetic Athena X-IFU observations. A\&A 618, 39 (2018). https://doi.org/10.1051/0004-6361/201833371

M. Rossetti, D. Eckert, S. De Grandi, F. Gastaldello, S. Ghizzardi, et al., Abell 2142 at large scales: An extreme case for sloshing? A\&A 556, 44 (2013). https://doi.org/10.1051/0004-6361/201321319

H.R. Russell, B.R. McNamara, A.C. Fabian, P.E.J. Nulsen, A.C. Edge, et al., ALMA observations of cold molecular gas filaments trailing rising radio bubbles in PKS 0745-191. MNRAS 458, 3134-3149 (2016). https://doi.org/10.1093/mnras/stw409

H.R. Russell, M. McDonald, B.R. McNamara, A.C. Fabian, P.E.J. Nulsen, et al., Alma Observations of Massive Molecular Gas Filaments Encasing Radio Bubbles in the Phoenix Cluster. ApJ 836, 130 (2017a). https://doi.org/10.3847/1538-4357/836/1/130

H.R. Russell, B.R. McNamara, A.C. Fabian, P.E.J. Nulsen, F. Combes, et al., Close en- 
trainment of massive molecular gas flows by radio bubbles in the central galaxy of Abell 1795. MNRAS 472, 4024-4037 (2017b). https://doi.org/10.1093/mnras/stx2255

M. Ruszkowski, S.P. Oh, Shaken and Stirred: Conduction and Turbulence in Clusters of Galaxies. ApJ 713, 1332-1342 (2010). https://doi.org/10.1088/0004-637X/713/2/1332

M. Ruszkowski, S.P. Oh, Galaxy motions, turbulence and conduction in clusters of galaxies. MNRAS 414, 1493-1507 (2011). https://doi.org/10.1111/j.1365-2966.2011.18482.x

M. Ruszkowski, M. Brüggen, M.C. Begelman, Cluster Heating by Viscous Dissipation of Sound Waves. ApJ 611, 158-163 (2004). https://doi.org/10.1086/422158

M. Ruszkowski, H.-Y.K. Yang, C.S. Reynolds, Cosmic-Ray Feedback Heating of the Intracluster Medium. ApJ 844, 13 (2017a). https://doi.org/10.3847/1538-4357/aa79f8

M. Ruszkowski, H.-Y.K. Yang, E. Zweibel, Global Simulations of Galactic Winds Including Cosmic-ray Streaming. ApJ 834, 208 (2017b). https://doi.org/10.3847/1538$4357 / 834 / 2 / 208$

D. Ryu, H. Kang, E. Hallman, T.W. Jones, Cosmological Shock Waves and Their Role in the Large-Scale Structure of the Universe. ApJ 593, 599-610 (2003). https://doi.org/10.1086/376723

I. Sakelliou, J.R. Peterson, T. Tamura, F.B.S. Paerels, J.S. Kaastra, et al., High resolution soft X-ray spectroscopy of M 87 with the reflection grating spectrometers on XMMNewton. A\&A 391, 903-909 (2002). https://doi.org/10.1051/0004-6361:20020900

P. Salomé, F. Combes, A.C. Edge, C. Crawford, M. Erlund, et al., Cold molecular gas in the Perseus cluster core. Association with X-ray cavity, $\mathrm{H} \alpha$ filaments and cooling flow. A\&A 454, 437-445 (2006). https://doi.org/10.1051/0004-6361:20054745

P. Salomé, F. Combes, Y. Revaz, D. Downes, A.C. Edge, A.C. Fabian, A very extended molecular web around NGC 1275. A\&A 531, 85 (2011). https://doi.org/10.1051/0004$6361 / 200811333$

J.S. Sanders, A.C. Fabian, Resonance scattering, absorption and off-centre abundance peaks in clusters of galaxies. MNRAS 370, 63-73 (2006). https://doi.org/10.1111/j.13652966.2006.10497.x

J.S. Sanders, A.C. Fabian, Deep Chandra and XMM-Newton X-ray observations of AWM 7 - I. Investigating X-ray surface brightness fluctuations. MNRAS 421, 726-742 (2012). https://doi.org/10.1111/j.1365-2966.2011.20348.x

J.S. Sanders, A.C. Fabian, Velocity width measurements of the coolest X-ray emitting material in the cores of clusters, groups and elliptical galaxies. MNRAS 429, 2727-2738 (2013). https://doi.org/10.1093/mnras/sts543

J.S. Sanders, A.C. Fabian, R.K. Smith, J.R. Peterson, A direct limit on the turbulent velocity of the intracluster medium in the core of Abell 1835 from XMM-Newton. MNRAS 402, 11-15 (2010a). https://doi.org/10.1111/j.1745-3933.2009.00789.x

J.S. Sanders, A.C. Fabian, K.A. Frank, J.R. Peterson, H.R. Russell, Deep highresolution X-ray spectra from cool-core clusters. MNRAS 402, 127-144 (2010b). https://doi.org/10.1111/j.1365-2966.2009.15902.x

C.L. Sarazin, C.M. Graney, Optical coronal emission lines from cooling flows in elliptical galaxies and galaxy clusters. ApJ 375, 532-543 (1991). https://doi.org/10.1086/170215

A. Sądowski, M. Gaspari, Kinetic and radiative power from optically thin accretion flows. MNRAS 468, 1398-1404 (2017). https://doi.org/10.1093/mnras/stx543

A.A. Schekochihin, S.C. Cowley, Turbulence, magnetic fields, and plasma physics in clusters of galaxies. Physics of Plasmas 13(5), 056501-056501 (2006). https://doi.org/10.1063/1.2179053

A.A. Schekochihin, S.C. Cowley, R.M. Kulsrud, G.W. Hammett, P. Sharma, Plasma Instabilities and Magnetic Field Growth in Clusters of Galaxies. ApJ 629, 139-142 (2005). https://doi.org/10.1086/431202

A.A. Schekochihin, S.C. Cowley, F. Rincon, M.S. Rosin, Magnetofluid dynamics of magnetized cosmic plasma: firehose and gyrothermal instabilities. MNRAS 405, 291-300 (2010). https://doi.org/10.1111/j.1365-2966.2010.16493.x

P. Schuecker, A. Finoguenov, F. Miniati, H. Böhringer, U.G. Briel, Probing turbulence in the Coma galaxy cluster. A\&A 426, 387-397 (2004). https://doi.org/10.1051/00046361:20041039

C. Shang, S.P. Oh, Probing gas motions in the intra-cluster medium: a mixture model approach. MNRAS 426, 3435-3454 (2012). https://doi.org/10.1111/j.1365- 
2966.2012.21897.x

C. Shang, S.P. Oh, Disentangling resonant scattering and gas motions in galaxy cluster emission line profiles. MNRAS 433, 1172-1184 (2013). https://doi.org/10.1093/mnras/stt790

P. Sharma, M. McCourt, E. Quataert, I.J. Parrish, Thermal instability and the feedback regulation of hot haloes in clusters, groups and galaxies. MNRAS 420, 3174-3194 (2012). https://doi.org/10.1111/j.1365-2966.2011.20246.x

A. Sheardown, E. Roediger, Y. Su, R.P. Kraft, T. Fish, et al., The Recent Growth History of the Fornax Cluster Derived from Simultaneous Sloshing and Gas Stripping: Simulating the Infall of NGC 1404. ApJ 865, 118 (2018). https://doi.org/10.3847/1538$4357 /$ aadc0f

X. Shi, D. Nagai, E.T. Lau, Multiscale analysis of turbulence evolution in the density-stratified intracluster medium. MNRAS 481, 1075-1082 (2018). https://doi.org/10.1093/mnras/sty2340

X. Shi, E. Komatsu, K. Nelson, D. Nagai, Analytical model for non-thermal pressure in galaxy clusters - II. Comparison with cosmological hydrodynamics simulation. MNRAS 448, 1020-1029 (2015). https://doi.org/10.1093/mnras/stv036

X. Shi, E. Komatsu, D. Nagai, E.T. Lau, Analytical model for non-thermal pressure in galaxy clusters - III. Removing the hydrostatic mass bias. MNRAS 455, 2936-2944 (2016). https://doi.org/10.1093/mnras/stv2504

R. Shibata, M. Ishida, N.Y. Yamasaki, T. Ohashi, K. Matsushita, et al., Distributions of the Temperature and Metal Abundance in the Virgo Cluster of Galaxies, in X-ray Astronomy 2000, ed. by R. Giacconi, S. Serio, L. Stella Astronomical Society of the Pacific Conference Series, vol. 234, 2001, p. 357

D. Sijacki, V. Springel, Hydrodynamical simulations of cluster formation with central AGN heating. MNRAS 366, 397-416 (2006). https://doi.org/10.1111/j.13652966.2005.09860.x

J. Silk, M.J. Rees, Quasars and galaxy formation. A\&A 331, 1-4 (1998)

A. Simionescu, N. Werner, A. Finoguenov, H. Böhringer, M. Brüggen, Metal-rich multi-phase gas in M 87. AGN-driven metal transport, magnetic-field supported multi-temperature gas, and constraints on non-thermal emission observed with XMM-Newton. A\&A 482 , 97-112 (2008). https://doi.org/10.1051/0004-6361:20078749

A. Simionescu, N. Werner, H. Böhringer, J.S. Kaastra, A. Finoguenov, et al., Chemical enrichment in the cluster of galaxies Hydra A. A\&A 493, 409-424 (2009). https://doi.org/10.1051/0004-6361:200810225

A. Simionescu, N. Werner, O. Urban, S.W. Allen, A.C. Fabian, et al., Large-scale Motions in the Perseus Galaxy Cluster. ApJ 757, 182 (2012). https://doi.org/10.1088/0004$637 \mathrm{X} / 757 / 2 / 182$

A. Simionescu, N. Werner, A. Mantz, S.W. Allen, O. Urban, Witnessing the growth of the nearest galaxy cluster: thermodynamics of the Virgo Cluster outskirts. MNRAS 469, 1476-1495 (2017). https://doi.org/10.1093/mnras/stx919

A. Simionescu, G. Tremblay, N. Werner, R.E.A. Canning, S.W. Allen, J.B.R. Oonk, ALMA observation of the disruption of molecular gas in M87. MNRAS 475, 3004-3009 (2018). https://doi.org/10.1093/mnras/sty047

W.B. Sparks, J.E. Pringle, M. Donahue, R. Carswell, M. Voit, et al., Discovery of C IV Emission Filaments in M87. ApJ 704, 20-24 (2009). https://doi.org/10.1088/0004$637 \mathrm{X} / 704 / 1 / \mathrm{L} 20$

Y. Su, R.P. Kraft, P.E.J. Nulsen, E. Roediger, W.R. Forman, et al., Capturing the 3D Motion of an Infalling Galaxy via Fluid Dynamics. ApJ 835, 19 (2017a). https://doi.org/10.3847/1538-4357/835/1/19

Y. Su, R.P. Kraft, E. Roediger, P.E.J. Nulsen, W.R. Forman, et al., Deep Chandra observations of NGC 1404: cluster plasma physics revealed by an infalling early-type galaxy. ApJ 834, 74 (2017b). https://doi.org/10.3847/1538-4357/834/1/74

C. Sugawara, M. Takizawa, K. Nakazawa, Suzaku Observation of the Radio Halo Cluster Abell 2319: Gas Dynamics and Hard X-Ray Properties. PASJ 61, 1293-1303 (2009). https://doi.org/10.1093/pasj/61.6.1293

M. Sun, M. Donahue, E. Roediger, P.E.J. Nulsen, G.M. Voit, et al., Spectacular X-ray Tails, Intracluster Star Formation, and ULXs in A3627. ApJ 708, 946-964 (2010). 
https://doi.org/10.1088/0004-637X/708/2/946

R.A. Sunyaev, E.M. Churazov, Millimeter-wavelength lines of heavy elements predicted from the hot gas in supernova remnants and galaxy clusters. Pisma v Astronomicheskii Zhurnal 10, 483-493 (1984)

R.A. Sunyaev, I.B. Zeldovich, The velocity of clusters of galaxies relative to the microwave background - The possibility of its measurement. MNRAS 190, 413-420 (1980). https://doi.org/10.1093/mnras/190.3.413

T. Tamura, K. Hayashida, S. Ueda, M. Nagai, Discovery of Gas Bulk Motion in the Galaxy Cluster Abell 2256 with Suzaku. PASJ 63, 1009-1017 (2011). https://doi.org/10.1093/pasj/63.sp3.S1009

T. Tamura, N.Y. Yamasaki, R. Iizuka, Y. Fukazawa, K. Hayashida, et al., Gas Bulk Motion in the Perseus Cluster Measured with Suzaku. ApJ 782, 38 (2014). https://doi.org/10.1088/0004-637X/782/1/38

T. Tanaka, H. Kunieda, M. Hudaverdi, A. Furuzawa, Y. Tawara, Non-Uniform Temperature Distribution in the Galaxy Clusters 2A 0335+096 and Abell 496 Observed by XMMNewton. PASJ 58, 703-718 (2006). https://doi.org/10.1093/pasj/58.4.703

X. Tang, E. Churazov, Sound wave generation by a spherically symmetric outburst and AGN feedback in galaxy clusters. MNRAS 468, 3516-3532 (2017). https://doi.org/10.1093/mnras/stx590

M. Tashiro, H. Maejima, K. Toda, R. Kelley, L. Reichenthal, et al., Concept of the X-ray Astronomy Recovery Mission, in Society of Photo-Optical Instrumentation Engineers (SPIE) Conference Series. Society of Photo-Optical Instrumentation Engineers (SPIE) Conference Series, vol. 10699, 2018, p. 1069922. https://doi.org/10.1117/12.2309455

P. Temi, A. Amblard, M. Gitti, F. Brighenti, M. Gaspari, et al., ALMA Observations of Molecular Clouds in Three Group-centered Elliptical Galaxies: NGC 5846, NGC 4636, and NGC 5044. ApJ 858, 17 (2018). https://doi.org/10.3847/1538-4357/aab9b0

G.R. Tremblay, C.P. O'Dea, S.A. Baum, R. Mittal, M.A. McDonald, et al., Far-ultraviolet morphology of star-forming filaments in cool core brightest cluster galaxies. MNRAS 451, 3768-3800 (2015). https://doi.org/10.1093/mnras/stv1151

G.R. Tremblay, F. Combes, J.B.R. Oonk, H.R. Russell, M.A. McDonald, et al., A Galaxyscale Fountain of Cold Molecular Gas Pumped by a Black Hole. ApJ 865, 13 (2018). https://doi.org/10.3847/1538-4357/aad6dd

M. Valentini, F. Brighenti, AGN-stimulated cooling of hot gas in elliptical galaxies. MNRAS 448, 1979-1998 (2015). https://doi.org/10.1093/mnras/stv090

M. van Dyke, An album of fluid motion (Parabolic Press, Stanford, 1982)

R.J. van Weeren, F. de Gasperin, H. Akamatsu, M. Brüggen, L. Feretti, et al., Diffuse Radio Emission from Galaxy Clusters. arXiv e-prints 1901.04496 (2019)

A.N. Vantyghem, B.R. McNamara, H.R. Russell, M.T. Hogan, A.C. Edge, et al., Molecular Gas Along a Bright $\mathrm{H} \alpha$ Filament in 2A 0335+096 Revealed by ALMA. ApJ 832, 148 (2016). https://doi.org/10.3847/0004-637X/832/2/148

F. Vazza, G. Brunetti, A. Kritsuk, R. Wagner, C. Gheller, M. Norman, Turbulent motions and shocks waves in galaxy clusters simulated with adaptive mesh refinement. A\&A 504, 33-43 (2009). https://doi.org/10.1051/0004-6361/200912535

F. Vazza, G. Brunetti, C. Gheller, R. Brunino, M. Brüggen, Massive and refined. II. The statistical properties of turbulent motions in massive galaxy clusters with high spatial resolution. A\&A 529, 17 (2011). https://doi.org/10.1051/0004-6361/201016015

J.C. Vernaleo, C.S. Reynolds, AGN Feedback and Cooling Flows: Problems with Simple Hydrodynamic Models. ApJ 645, 83-94 (2006). https://doi.org/10.1086/504029

R. Vijayaraghavan, C. Sarazin, The Evaporation and Survival of Cluster Galaxies' Coronae. II. The Effectiveness of Anisotropic Thermal Conduction and Survival of Stripped Galactic Tails. ApJ 848, 63 (2017a). https://doi.org/10.3847/1538-4357/aa8bb3

R. Vijayaraghavan, C. Sarazin, The Evaporation and Survival of Cluster Galaxy Coronae. I. The Effectiveness of Isotropic Thermal Conduction Including Saturation. ApJ 841, 22 (2017b). https://doi.org/10.3847/1538-4357/aa706d

A. Vikhlinin, M. Markevitch, S.S. Murray, A Moving Cold Front in the Intergalactic Medium of A3667. ApJ 551, 160-171 (2001a). https://doi.org/10.1086/320078

A. Vikhlinin, M. Markevitch, S.S. Murray, Chandra Estimate of the Magnetic Field Strength near the Cold Front in A3667. ApJ 549, 47-50 (2001b). https://doi.org/10.1086/319126 
G.M. Voit, A Role for Turbulence in Circumgalactic Precipitation. ApJ 868, 102 (2018). https://doi.org/10.3847/1538-4357/aae8e2

G.M. Voit, M. Donahue, J.D. Slavin, Emission lines from condensing intracluster gas. ApJS 95, 87-105 (1994). https://doi.org/10.1086/192095

G.M. Voit, M. Donahue, G.L. Bryan, M. McDonald, Regulation of star formation in giant galaxies by precipitation, feedback and conduction. Nature 519, 203-206 (2015). https://doi.org/10.1038/nature14167

S.A. Walker, A.C. Fabian, J.S. Sanders, Large-scale gas sloshing out to half the virial radius in the strongest cool core REXCESS galaxy cluster, RXJ2014.8-2430. MNRAS 441, 31-35 (2014). https://doi.org/10.1093/mnrasl/slu040

S.A. Walker, J.S. Sanders, A.C. Fabian, Constraining gas motions in the Centaurus cluster using X-ray surface brightness fluctuations and metal diffusion. MNRAS 453, 3699-3705 (2015). https://doi.org/10.1093/mnras/stv1929

S.A. Walker, J.S. Sanders, A.C. Fabian, What fraction of the density fluctuations in the Perseus cluster core is due to gas sloshing rather than AGN feedback? MNRAS 481, 1718-1725 (2018b). https://doi.org/10.1093/mnras/sty2390

S.A. Walker, J. Hlavacek-Larrondo, M. Gendron-Marsolais, A.C. Fabian, H. Intema, et al., Is there a giant Kelvin-Helmholtz instability in the sloshing cold front of the Perseus cluster? MNRAS 468, 2506-2516 (2017). https://doi.org/10.1093/mnras/stx640

S.A. Walker, J. ZuHone, A. Fabian, J. Sanders, The split in the ancient cold front in the Perseus cluster. Nature Astronomy 2, 292-296 (2018a). https://doi.org/10.1038/s41550018-0401-8

S. Walker, A. Simionescu, D. Nagai, N. Okabe, D. Eckert, et al., The Physics of Galaxy Cluster Outskirts. Space Sci. Rev. 215, 7 (2019). https://doi.org/10.1007/s11214-018$0572-8$

N. Werner, I. Zhuravleva, E. Churazov, A. Simionescu, S.W. Allen, et al., Constraints on turbulent pressure in the X-ray haloes of giant elliptical galaxies from resonant scattering. MNRAS 398, 23-32 (2009). https://doi.org/10.1111/j.1365-2966.2009.14860.x

N. Werner, J.B.R. Oonk, M. Sun, P.E.J. Nulsen, S.W. Allen, et al., The origin of cold gas in giant elliptical galaxies and its role in fuelling radio-mode AGN feedback. MNRAS 439, 2291-2306 (2014). https://doi.org/10.1093/mnras/stu006

N. Werner, J.A. ZuHone, I. Zhuravleva, Y. Ichinohe, A. Simionescu, et al., Deep Chandra observation and numerical studies of the nearest cluster cold front in the sky. MNRAS 455, 846-858 (2016). https://doi.org/10.1093/mnras/stv2358

N. Werner, B.R. McNamara, E. Churazov, E. Scannapieco, Hot Atmospheres, Cold Gas, AGN Feedback and the Evolution of Early Type Galaxies: A Topical Perspective. Space Sci. Rev. 215, 5 (2019). https://doi.org/10.1007/s11214-018-0571-9

J. Wiener, C. Pfrommer, S.P. Oh, Cosmic ray-driven galactic winds: streaming or diffusion? MNRAS 467, 906-921 (2017). https://doi.org/10.1093/mnras/stx127

J. Wilms, T. Brand, D. Barret, T. Beuchert, J.-W. den Herder, et al., ATHENA end-to-end simulations, in Space Telescopes and Instrumentation 2014: Ultraviolet to Gamma Ray. Proc. SPIE, vol. 9144, 2014, p. 91445. https://doi.org/10.1117/12.2056347

R.A. Wood, C. Jones, M.E. Machacek, W.R. Forman, A. Bogdan, et al., The Infall of the Virgo Elliptical Galaxy M60 toward M87 and the Gaseous Structures Produced by Kelvin-Helmholtz Instabilities. ApJ 847(1) (2017). https://doi.org/10.3847/1538$4357 /$ aa 8723

H. Xu, S.M. Kahn, J.R. Peterson, E. Behar, F.B.S. Paerels, et al., High-Resolution Observations of the Elliptical Galaxy NGC 4636 with the Reflection Grating Spectrometer on Board XMM-Newton. ApJ 579, 600-606 (2002). https://doi.org/10.1086/342828

L. Yan, J.G. Cohen, Search for Coronal Emission Lines in Cooling Flow Clusters with the Keck 10 Meter Telescope. ApJ 454, 44 (1995). https://doi.org/10.1086/176462

C. Zhang, E. Churazov, A.A. Schekochihin, Generation of internal waves by buoyant bubbles in galaxy clusters and heating of intracluster medium. MNRAS 478, 4785-4798 (2018). https://doi.org/10.1093/mnras/sty1269

I.V. Zhuravleva, E.M. Churazov, S.Y. Sazonov, R.A. Sunyaev, K. Dolag, Resonant scattering in galaxy clusters for anisotropic gas motions on various spatial scales. Astronomy Letters 37, 141-153 (2011). https://doi.org/10.1134/S1063773711010087

I. Zhuravleva, E. Churazov, A. Kravtsov, R. Sunyaev, Constraints on the ICM velocity 
power spectrum from the X-ray lines width and shift. MNRAS 422, 2712-2724 (2012). https://doi.org/10.1111/j.1365-2966.2012.20844.x

I. Zhuravleva, E. Churazov, A. Kravtsov, E.T. Lau, D. Nagai, R. Sunyaev, Quantifying properties of ICM inhomogeneities. MNRAS 428, 3274-3287 (2013a). https://doi.org/10.1093/mnras/sts275

I. Zhuravleva, E. Churazov, R. Sunyaev, S. Sazonov, S.W. Allen, et al., Resonant scattering in the Perseus Cluster: spectral model for constraining gas motions with Astro-H. MNRAS 435, 3111-3121 (2013b). https://doi.org/10.1093/mnras/stt1506

I. Zhuravleva, E.M. Churazov, A.A. Schekochihin, E.T. Lau, D. Nagai, et al., The Relation between Gas Density and Velocity Power Spectra in Galaxy Clusters: Qualitative Treatment and Cosmological Simulations. ApJ 788, 13 (2014a). https://doi.org/10.1088/2041-8205/788/1/L13

I. Zhuravleva, E. Churazov, A.A. Schekochihin, S.W. Allen, P. Arévalo, et al., Turbulent heating in galaxy clusters brightest in X-rays. Nature 515, 85-87 (2014b). https://doi.org/10.1038/nature13830

I. Zhuravleva, E. Churazov, P. Arévalo, A.A. Schekochihin, S.W. Allen, et al., Gas density fluctuations in the Perseus Cluster: clumping factor and velocity power spectrum. MNRAS 450, 4184-4197 (2015). https://doi.org/10.1093/mnras/stv900

I. Zhuravleva, E. Churazov, P. Arévalo, A.A. Schekochihin, W.R. Forman, et al., The nature and energetics of AGN-driven perturbations in the hot gas in the Perseus Cluster. MNRAS 458, 2902-2915 (2016). https://doi.org/10.1093/mnras/stw520

I. Zhuravleva, S.W. Allen, A. Mantz, N. Werner, Gas Perturbations in the Cool Cores of Galaxy Clusters: Effective Equation of State, Velocity Power Spectra, and Turbulent Heating. ApJ 865, 53 (2018). https://doi.org/10.3847/1538-4357/aadae3

E. Zinger, A. Dekel, Y. Birnboim, D. Nagai, E. Lau, A.V. Kravtsov, Cold fronts and shocks formed by gas streams in galaxy clusters. MNRAS 476, 56-70 (2018). https://doi.org/10.1093/mnras/sty136

J.A. Zuhone, E. Roediger, Cold fronts: probes of plasma astrophysics in galaxy clusters. Journal of Plasma Physics 82(3), 535820301 (2016). https://doi.org/10.1017/S0022377816000544

J.A. ZuHone, M. Markevitch, M. Ruszkowski, D. Lee, Cold Fronts and Gas Sloshing in Galaxy Clusters with Anisotropic Thermal Conduction. ApJ 762, 69 (2013). https://doi.org/10.1088/0004-637X/762/2/69

J.A. ZuHone, E.D. Miller, A. Simionescu, M.W. Bautz, Simulating Astro-H Observations of Sloshing Gas Motions in the Cores of Galaxy Clusters. ApJ 821, 6 (2016) https://doi.org/10.3847/0004-637X/821/1/6

J.A. ZuHone, E.D. Miller, E. Bulbul, I. Zhuravleva, What Do the Hitomi Observations Tell Us About the Turbulent Velocities in the Perseus Cluster? Probing the Velocity Field with Mock Observations. ApJ 853, 180 (2018). https://doi.org/10.3847/1538$4357 /$ aaa4b3 